\title{
Organ-Specific Branching Morphogenesis
}

\author{
Christine Lang ${ }^{1,2 \dagger}$, Lisa Conrad ${ }^{1,2 \dagger}$ and Dagmar Iber ${ }^{1,2 *}$ \\ ${ }^{1}$ Department of Biosystems, Science and Engineering, ETH Zürich, Basel, Switzerland, ${ }^{2}$ Swiss Institute of Bioinformatics, \\ Basel, Switzerland
}

A common developmental process, called branching morphogenesis, generates the epithelial trees in a variety of organs, including the lungs, kidneys, and glands. How branching morphogenesis can create epithelial architectures of very different shapes and functions remains elusive. In this review, we compare branching morphogenesis and its regulation in lungs and kidneys and discuss the role of signaling pathways, the

OPEN ACCESS

Edited by:

Zuoren Yu,

Tongji University, China

Reviewed by:

lan Smyth,

Monash University, Australia

Rajprasad Loganathan,

Johns Hopkins University,

United States

David Warburton,

Saban Research Institute, Children's

Hospital Los Angeles, United States

*Correspondence:

Dagmar lber

dagmar.iber@bsse.ethz.ch

tThese authors have contributed equally to this work and share first authorship

Specialty section:

This article was submitted to Cell Growth and Division, a section of the journal

Frontiers in Cell and Developmental

Biology

Received: 23 February 2021 Accepted: 06 May 2021

Published: 07 June 2021

Citation:

Lang $C$, Conrad $L$ and Iber D (2021) Organ-Specific Branching Morphogenesis.

Front. Cell Dev. Biol. 9:671402. doi: 10.3389/fcell.2021.671402 mesenchyme, the extracellular matrix, and the cytoskeleton as potential organ-specific determinants of branch position, orientation, and shape. Identifying the determinants of branch and organ shape and their adaptation in different organs may reveal how a highly conserved developmental process can be adapted to different structural and functional frameworks and should provide important insights into epithelial morphogenesis and developmental disorders.

Keywords: branching morphogenesis, lung, kidney, branch distance, branch angle, branch shape, turing pattern, tissue mechanics

\section{INTRODUCTION}

Highly branched tubular structures are a common architectural motif of many organs, such as lungs, kidneys, and glands, that require a high surface to volume ratio for their function ( $\mathrm{Lu}$ and Werb, 2008; Ochoa-Espinosa and Affolter, 2012; Iber and Menshykau, 2013; Spurlin and Nelson, 2016; Goodwin and Nelson, 2020). The central structure of branched organs is composed of tightly associated epithelial cells surrounded by rather loosely connected mesenchymal cells. The developmental process during which epithelial buds branch into the surrounding mesenchyme and thereby construct a complex epithelial tree is called branching morphogenesis. The biochemical and mechanical interactions between epithelium and mesenchyme that regulate branching morphogenesis have been studied in great detail, and the key regulatory factors that control branching morphogenesis are largely well known (Shah, 2004; Warburton et al., 2005; Jones et al., 2021). How the commonly used principle of branching morphogenesis can create organs of different shapes and functions, nonetheless, remains elusive.

In the following, we will focus on the regulation of branching processes in the lung and kidney because both organs share many similarities with regard to branching morphogenesis, but still have a very different organ shape and function. For both organs, branching is suggested to be highly stereotyped, which does not apply to glandular organs like pancreas, prostate, thyroid, salivary and mammary gland (Lu and Werb, 2008; Villasenor et al., 2010; Wang et al., 2017). In contrast to lung and kidney, branching patterns in the mammary gland or prostate are substantially affected by hormones (Sternlicht et al., 2006; Prins and Putz, 2008). Epithelial branching in lungs and kidneys is accompanied by overall organ growth, while the stroma in the mammary gland is 
already established and does not substantially change in size when the epithelium starts to invade it (Sternlicht et al., 2006).

Branching morphogenesis has been studied in model organisms other than the mouse, rat and chick (Affolter and Caussinus, 2008; Bracken et al., 2008; Horowitz and Simons, 2008; Shifley et al., 2012; Rankin et al., 2015). While orthologs of many genes involved in branching have similar roles in other species, these are not within the scope of this review.

\section{BRANCHING MORPHOGENESIS IN LUNG AND KIDNEY}

Branching morphogenesis of the mouse lung primarily occurs during the pseudoglandular stage of lung development between embryonic day (E) 10.5 and E16.5 (Herriges and Morrisey, 2014; Schittny, 2017). At around E10.5, after initial bronchial formation, the lung lobes have started to form, with the right lung consisting of four lobes and the left lung remaining as a single lobe, and then continue to repeatedly elongate and branch into the surrounding mesenchyme (Cardoso and Lü, 2006; Warburton et al., 2010). After 13-17 branch generations, the lung tree consists of more than 5,000 branches (Metzger et al., 2008). Kidney branching morphogenesis in the mouse starts around E11.5 after the ureteric bud (UB) has invaded the metanephric mesenchyme (MM) and established the characteristic T-shape (McMahon, 2016). Subsequent repeated rounds of around 9-12 generations of epithelial branching and elongation transform the UB into a complex tree with around 2,600 branch and tip segments at E16.5 (Cebrián et al., 2004; Short et al., 2014).

Most branched organs require the strict modulation of at least one receptor tyrosine kinase (RTK) signaling pathway whose modulation is strictly controlled ( $\mathrm{Lu}$ and Werb, 2008). The associated ligands are usually expressed in the mesenchyme, while the respective receptors are produced in the epithelium. Fibroblast growth factor 10 (FGF10) and glial cell line-derived neurotrophic factor (GDNF) signaling are essential for branching in the lung and kidney, respectively (Moore et al., 1996; Pichel et al., 1996; Sánchez et al., 1996; Min et al., 1998; Sekine et al., 1999). FGF10 signals through its receptor fibroblast growth factor receptor 2b (FGFR2b), and GDNF via its receptor rearranged during infection (RET) and co-receptor GDNF family receptor $\alpha(G F R \alpha)$ (Jing et al., 1996; Treanor et al., 1996; Ohuchi et al., 2000). Fgf10 ${ }^{-/}$and Fgfr2 $\mathrm{b}^{-/-}$mice do not develop lungs and die at birth (Moerlooze et al., 2000; Ohuchi et al., 2000), while the absence of GDNF or its receptors results in kidney agenesis (Schuchardt et al., 1994, 1996; Moore et al., 1996; Pichel et al., 1996; Sánchez et al., 1996; Cacalano et al., 1998). FGF10 and GDNF both induce extracellular-signal-regulated kinase (ERK) and phosphoinositide 3-kinase (PI3K) signaling and converge on the transcription factors ETS translocation variant 4/5 (Etv4/5) to promote branching morphogenesis (Fisher et al., 2001; Tang et al., 2002; Liu et al., 2003; Lu et al., 2009; Zhu and Nelson, 2012; Herriges et al., 2015). Another important shared signaling pathway is wingless-related integration site $(\mathrm{Wnt}) / \beta$-catenin (De Langhe et al., 2005; Hashimoto et al., 2012; Sarin et al., 2014;
Ostrin et al., 2018). FGF10 signaling plays an important role during kidney branching as well. Thus, kidneys from $\mathrm{Fgf10^{-/- }}$ and $\mathrm{Fgfr} 2^{-/-}$mice are characterized by reduced size and dysplasia (Ohuchi et al., 2000; Zhao et al., 2004; Michos et al., 2010). Moreover, treatment of embryonic kidney cultures with soluble FGFR2b inhibits branching (Qiao et al., 2001). In the absence of Sprouty1, an ERK signaling inhibitor, FGF10 rescues the $G d n f^{-/-}$phenotype and is therefore considered to have an at least partly redundant function to GDNF (Michos et al., 2010). GDNF and its receptors are expressed in the lung as well, but only from E13.5 onward, and neither exogenous GDNF nor a GDNF-blocking antibody affects lung development (Towers et al., 1998).

Both organs show specific expression patterns in epithelium and mesenchyme for FGF10 and GDNF and the respective receptors. During lung branching morphogenesis, Fgf10 is expressed in spatially restricted domains in the submesothelial mesenchyme around distal epithelial buds, while the expression of its receptor $F g f r 2 b$ is localized to distal lung epithelia (Figure 1A; Bellusci et al., 1997). At E13.5, Gdnf is present throughout the lung mesenchyme, while Ret is detected throughout the epithelium and its co-receptor Gfro is expressed exclusively in the proximal epithelium and mesenchyme (Towers et al., 1998). In the kidney, Fgf10 is expressed throughout the $\mathrm{MM}$ at E11.0 and becomes enriched in the cap mesenchyme (CM) by E12.5, while Fgfr2 expression is present in the UB epithelium (Figure 1B; Zhao et al., 2004; Michos et al., 2010; Sanna-Cherchi et al., 2013; Trueb et al., 2013; Brunskill et al., 2014). Gdnf is expressed in the CM, which surrounds the branching UB tips, and in the kidney stroma (Figure 1B; Hellmich et al., 1996; Magella et al., 2018). Ret is expressed exclusively in the UB and becomes restricted to bud tips through a cell sorting mechanism as branching morphogenesis progresses, while its co-receptor Gfrol is expressed in both the UB and the MM (Figure 1B; Pachnis et al., 1993; Riccio et al., 2016; Rutledge et al., 2017). The spatially restricted expression domains of FGF10 in the lung and RET in the kidney suggest that distinct expression patterns play an important role in regulating organ-specific branching. However, uniform Fgf10 expression does not abrogate lung branching morphogenesis but regulates epithelial lineage commitment (Volckaert et al., 2013). Similarly, expression of Ret throughout the UB instead of only at the tips does not inhibit branching but causes retardation of kidney development (Srinivas et al., 1999).

Fibroblast growth factor 10 and GDNF signaling are regulated by several stimulatory and inhibitory factors, including $\mathrm{SHH}$, Sprouty, WNT, and bone morphogenetic protein (BMP)/transforming growth factor- $\beta$ (TGF- $\beta$ ) signaling (Costantini and Kopan, 2010; Yuan et al., 2018). FGF10 signaling in the lung triggers the expression of Shh in the epithelium which in turn represses Fgf10 expression in the mesenchyme (Figure 1C; Bellusci et al., 1997; Lebeche et al., 1999). This negative feedback loop between FGF10 and $\mathrm{SHH}$ regulates the spatial-temporal dynamics of Fgf10 expression in the lung mesenchyme. GDNF signaling in the kidney upregulates Wnt11 expression in the epithelium, enhancing Gdnf expression in the mesenchyme (Figure 1D; Majumdar et al., 2003). 


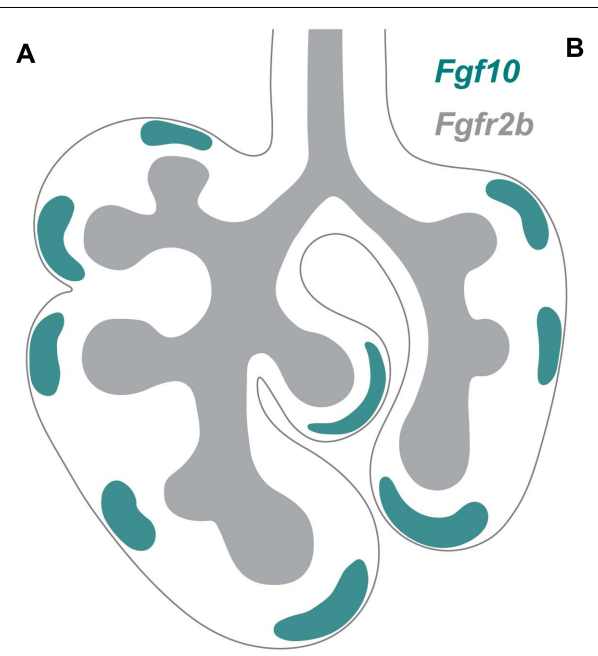

B

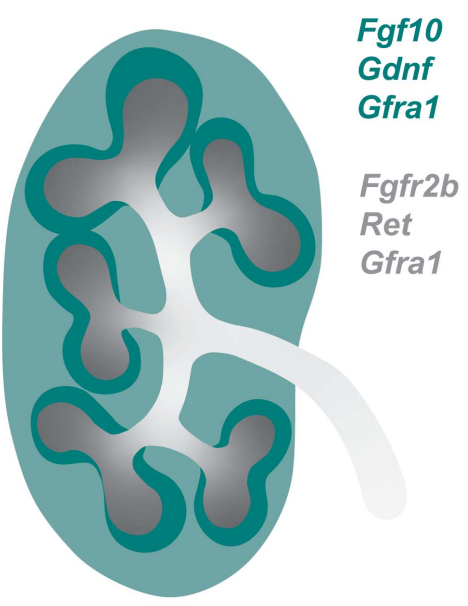

C

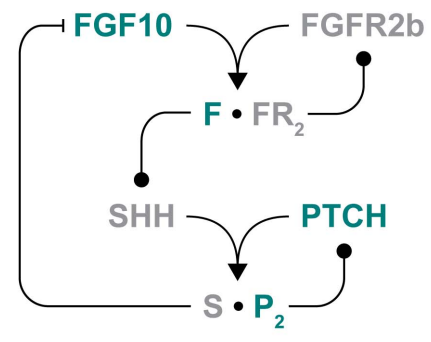

D

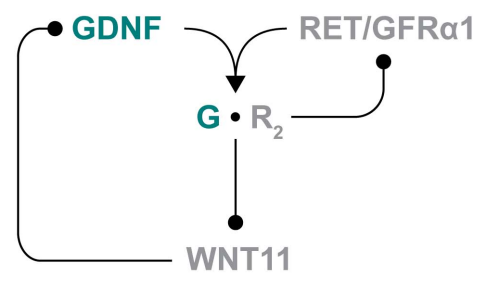

E

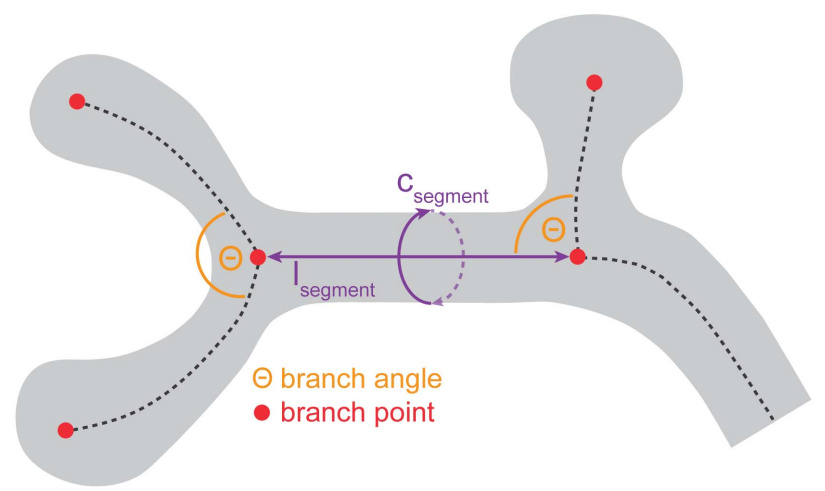

FIGURE 1 | Branching morphogenesis. (A) Gene expression domains of Fgf10 and its receptor Fgfr2b in the E11.5 mouse lung. Fgf10 is expressed in a spotty pattern in the submesothelial mesenchyme (green), while Fgfr2b is expressed in the epithelium (gray). (B) Gene expression domains of Gdnf and Fgf10 and their receptors in the E12.5 murine kidney. Gdnf is expressed in both the cap mesenchyme (dark green) and the stroma (light green), while Fgf10 is expressed in the cap mesenchyme. Fgfr2b is expressed in the ureteric bud (UB), while Ret expression is restricted to UB tips (dark gray). The Ret co-receptor Gfra1 is expressed both in the metanephric mesenchyme and the ureteric bud. (C) Core signaling network in lung branching morphogenesis. FGF10 signals via FGFR2b which enhances Shh expression. SHH signaling negatively regulates Fgf10 expression. (D) Core signaling network in kidney branching morphogenesis. GDNF signals via RET and GFR $\alpha 1$ which enhances Wnt11 expression. WNT11 signaling positively regulates Gdnf expression. (E) Hallmarks of branching: The morphology of branched epithelial trees is governed by the branching mode/sequence (branch points; red), the length and circumference of branches (purple) and by the branching angle (orange).

This positive feedback between GDNF and WNT11 plays an important role in the regulation of tip packaging in the kidney (Menshykau et al., 2019).

A further distinction can be made between the distal branch tips and the proximal stalks of the branching epithelium. Tips and stalks are characterized by distinct sets of expressed genes, some of which are specific to the lung or UB tip, while others are expressed in both organs (lung tip genes summarized in Nikolić et al., 2017; Rutledge et al., 2017).

\section{HALLMARKS OF BRANCHING}

During organogenesis, lungs and kidneys generate highly arborized epithelial networks from simple epithelial organ bud primordia (Herriges and Morrisey, 2014; McMahon, 2016; Schittny, 2017). The organs differ in the extent and rate of branching, i.e., the number of created branches after a certain amount of time, as well as in the distance, orientation, and branch shape (Figure 1E). How these characteristics are regulated 
during branching morphogenesis in different organs is an important field of study.

Branching processes are typically studied using microscopy imaging data. Organ explant cultures have proven to be a valuable method to monitor branching processes in $2 \mathrm{D}$ over a desired period of time (del Moral and Warburton, 2010; Michos, 2012). This method, however, is not able to capture branching aspects in $3 \mathrm{D}$ and is reported to recapitulate in vivo branching only to a certain extent due to emerging artifacts (Watanabe and Costantini, 2004; Short and Smyth, 2016). 3D imaging provides comprehensive reconstructions of branched trees (Short et al., 2010). As live imaging of organs in 3D over long periods of time is still limited, only snapshots of certain developmental stages are available so far. The quantitative analysis of either $2 \mathrm{D}$ or $3 \mathrm{D}$ imaging data on branching morphogenesis is challenging due to the high complexity of branched structures. Image processing and analysis methods have now advanced over the last years to enable comprehensive studies on branch position, branch orientation, and branch shape (Short and Smyth, 2016). All these characteristics are essential to describe and compare epithelial tree architectures (Figure 1E). In this section, we highlight developmental mechanisms that are known to contribute to the regulation of these branching hallmarks.

\section{Branch Position}

\section{The Sequence and Stereotypy of Branch Emergence}

To arrive at an elaborately branched epithelial tree from a simple organ anlage, new branches need to emerge. Generally, new branches can be formed by the splitting of an existing tip in two or more daughter branches or by the formation of a new bud on the side of an existing tube. The sequence in which new branch points emerge is a significant determinant of the final branched tree and differs markedly between the lung and the kidney (Metzger et al., 2008; Short et al., 2013). The lung epithelium forms new branches by lateral branching and through tip bifurcations in planar or orthogonal orientation (Figure 2A; Metzger et al., 2008; Short et al., 2013). In the kidney, new branches form through tip bifurcations and sometimes trifurcations, which, however, resolve into bifurcations during continued development (Figure 2A; Short et al., 2014). Although the direction in which branches can grow is constrained by the flattened morphology of the organ, lungs and kidneys cultured in ex vivo organ culture systems form new branches using the same branching modes as their in vivo counterparts. However, in kidney explants, lateral branching events at "trunk" segments are sometimes observed (Lin et al., 2003; Watanabe and Costantini, 2004).

The branching program of the murine embryonic lung has been characterized in great detail by Metzger et al. (2008) suggesting that lung branching is highly stereotyped. The 3D reconstructions of E14.5 lungs from Short et al. (2013) correspond well with the previously proposed branching trees, although substantially more variations in branch positions and directions are observed, suggesting that stereotypy in lung branching is generally robust, but also allows for deviations on a finer scale. The relaxation of the branching stereotypy beyond the first rounds of branching has been related to dynamic changes in the growth of the mesenchyme with branches adapting to the available space, highlighting the importance of coupled epithelial-mesenchymal growth (Blanc et al., 2012). A similar picture emerges for the kidney since internal branches appear to emerge in a highly stereotypical manner, while branches at the periphery show a higher degree of variations. This finding is based on subdividing the ureteric tree into several anterior and posterior clades, according to the first branches formed at E12.5, which show comparable branching patterns and complexity between individual organs and potentially manifest a comparable structural stereotypy as the different lobes in the lung (Short et al., 2014; Lefevre et al., 2017).

\section{The Mesenchyme as a Modulator of Branching Patterns}

Branching morphogenesis depends on inductive signals and complex, reciprocal interactions between the branching epithelium and the surrounding mesenchyme. The branching pattern of the lung epithelium is coupled to the shape of the surrounding mesenchyme. Dynamic changes in the direction of mesenchyme growth guide the specification of branch points and subsequent branch outgrowth. For example, domain branches are formed when the mesenchyme growth changes in a perpendicular direction during lung development (Blanc et al., 2012).

Tissue recombination experiments in the salivary gland and the lung have demonstrated the ability of the mesenchyme to support continued branching of the UB (Grobstein, 1955; Kispert et al., 1996; Sainio et al., 1997). Notably, only bronchial mesenchyme supports continued branching of the lung epithelium (Spooner and Wessells, 1970; Alescio and Dani, 1971). While the lung epithelium is viable and able to form single buds when recombined with mesenchyme from the submandibular, salivary and mammary gland, as well as with mesenchyme from other sources (Spooner and Wessells, 1970; Duernberger and Kratochwil, 1980), further branching is only observed when the mesenchyme is renewed (Lawson, 1983).

The identification of mesenchymal-derived morphogens required for branching morphogenesis of the lung and the kidney epithelium has made it possible to culture branching isolated epithelia embedded in extracellular matrix (ECM) gels. While requiring the appropriate growth factors, this has demonstrated an intrinsic ability of the epithelium to form new branches (Nogawa and Ito, 1995; Bellusci et al., 1997; Qiao et al., 1999; Varner et al., 2015; Conrad et al., 2021). However, the branching pattern in these isolated cultures appears very different from the in vivo tree as the formation of new branches eventually stops, highlighting the importance of the mesenchyme for the modulation of the branching pattern and the maintenance of cellular niches.

A detailed morphometric characterization of the branching patterns of recombined lung and kidney tissue has shown that the lung mesenchyme "reprograms" the UB to adapt a branching pattern that is typical for the lung. This demonstrates the importance of the mesenchyme in specifying the branching mode and in establishing organ-specific branching patterns (Figure 2B; Lin et al., 2003). Similar reprogramming of 


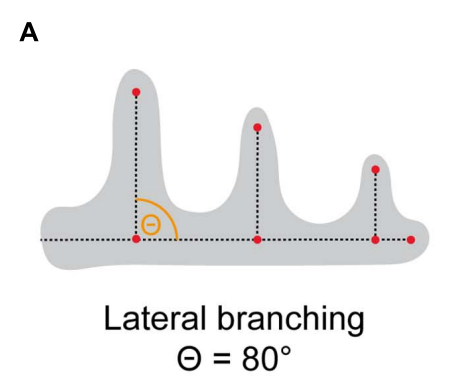

B Tissue separation

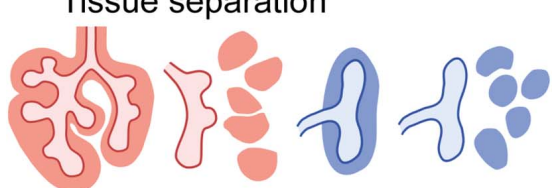

Tissue recombination
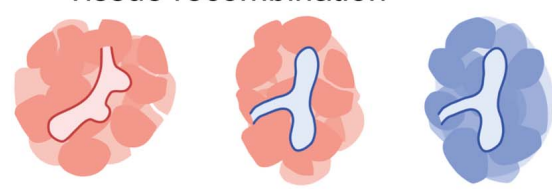

Resulting branching pattern

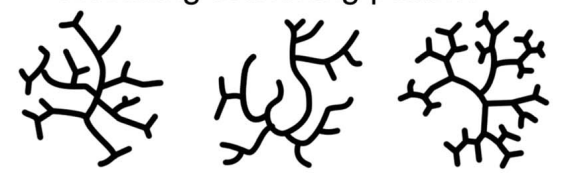

D

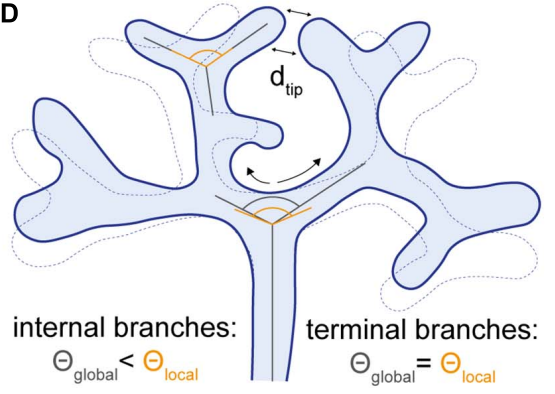

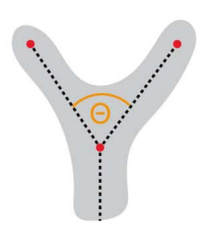

Bifurcations $\Theta=100-115^{\circ}$

C

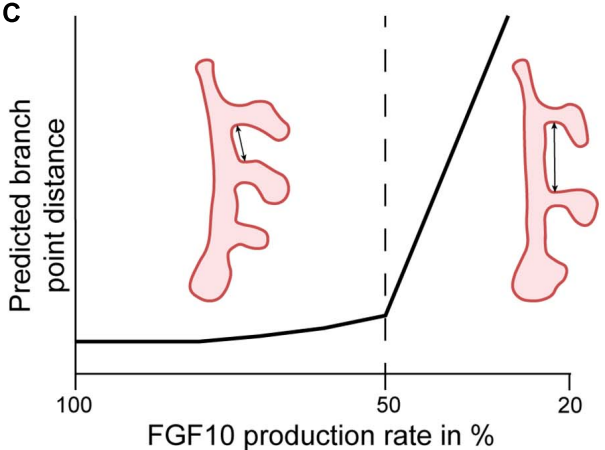

E

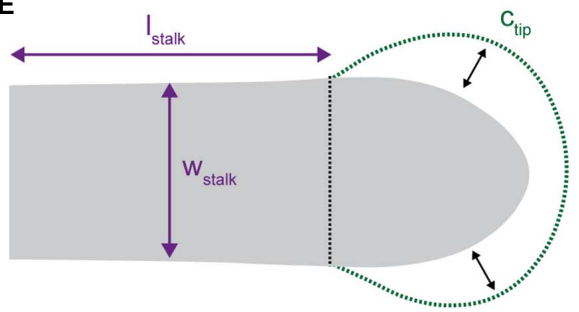

Mitosis spindle orientation Cell shape \& tension

Fluid flow/shear stress

Transport

FGF10/GDNF signalling Vasculature

ECM remodelling

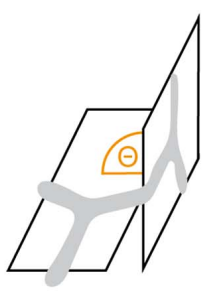

Rotations

$\Theta=60-65^{\circ}$

FIGURE 2 | Regulation principles of branching hallmarks in lung and kidney morphogenesis. (A) Branching modes used by lungs and kidneys. Lateral branches in the lung are oriented at an angle of around $80^{\circ}$. In both organs, bifurcations occur at a divergence angle of around $100-115^{\circ}$, while rotations of bifurcation events show a dihedral angle of around 60-65 . (B) Mesenchyme as pattern modulator. Separation of mesenchymal and epithelial tissue and subsequent re-arrangement of the mesenchyme around the epithelium destroys any potential mesenchymal pre-patterning. In homotypic recombination experiments, the respective organ-specific branching pattern is maintained, while in heterotypic recombination experiments lung mesenchyme "reprograms" the ureteric bud to adapt a lung-like branching pattern. (C) Regulation of branch point distance in the lung. Fgf10 hypomorphic lungs with a reduction of FGF10 expression by $55 \%$ exhibit a wider spacing of the first three lateral branches of the left lung lobe (right lung scheme), while other allelic combinations with a milder reduction in Fgf10 expression do not differ from wild type lungs (left lung scheme). The lung schemes were reproduced from Ramasamy et al. (2007). Accordingly, the ligand-receptor-based Turing mechanism predicts that the spacing of branch tips depends on the rate of ligand expression and is only affected if this rate falls below a certain threshold (dashed line). The graph was reproduced from Celliere et al. (2012). (D) Branch angle remodeling in the kidney. Local bifurcation angles (starting direction of daughter branches) are relatively constant, while global bifurcation angles (direction relative to terminal branch point) show spatial and temporal dynamics. Compressive remodeling of internal branches leads to an increased curvature of these internal branches. Concurrently, terminal branches move closer toward each other which reduces the inter-tip distance $d_{\text {tip }}$, thereby promoting tip packaging. (E) Branch shape regulation. The shape of branch stalks and tips are regulated differently. The branches in lungs and kidneys show anisotropic growth, meaning that the increase in stalk length $I_{\text {stalk }}$ is larger than in stalk width $w_{\text {stalk, }}$ due to a biased mitosis spindle orientation. Fluid flow and resulting shear stress is able to explain biased elongation in lungs and kidneys. Branch tip shape is regulated by several factors, such as signaling interactions, ECM remodeling or cell tension dynamics, which are highly interconnected. Perturbation of these shape determinants primarily leads to dilated buds characterized by an increased tip circumference $c_{\text {tip }}$ and the absence of cleft formation.

organ-specific branching is observed when the mammary epithelium is recombined with salivary gland mesenchyme, suggesting a broader context in which the mesenchyme can specify epithelial branching patterns (Kratochwil, 1969; Sakakura et al., 1976). In tissue recombination experiments, the mesenchyme typically gets cut up into pieces and placed around 
the epithelium, scrambling any pre-patterning. The use of organspecific branching modes of lungs and kidneys is maintained in homotypic recombination experiments (Figure 2B; Lin et al., 2003), suggesting that new branch points are formed spontaneously from regulatory interactions.

\section{A Ligand-Receptor-Based Turing Mechanism Regulates Branch Point Specification and Distance}

The question of how the patterning and branching of epithelial tubes are regulated has inspired many different models for branching morphogenesis (reviewed in Iber and Menshykau, 2013; Lang et al., 2018). What kind of mechanism could result in highly stereotyped branching, independent of a pre-patterning, and at the same time allow for variations in the branching pattern and adaptations to available space in the mesenchyme? Alan Turing's work on pattern formation in biological systems, which showed that self-organized symmetry breaks could result from diffusion-driven instabilities of morphogens, laid the foundation for computational studies on pattern formation (Turing, 1952; Gierer and Meinhardt, 1972). Our group has shown that the ligand-receptor interactions between FGF10 and FGFR2b, as well as between SHH and its receptor Patched-1 (PTCH) in the lung (Menshykau et al., 2012, 2014; Kurics et al., 2014) and between GDNF and RET in the kidney (Menshykau and Iber, 2013; Menshykau et al., 2019) can give rise to Turing patterns (Figures 1C,D). First modeled on an idealized tube domain, the interactions between FGF10, $\mathrm{SHH}$, and PTCH, as well as GDNF/RET signaling in a positive feedback loop with WNT11, both give rise to Turing patterns that, depending on the parameter values of the model, represent distinct branch modes in the lung and the kidney, namely tip bifurcation, trifurcation, and lateral branching (Menshykau et al., 2012; Menshykau and Iber, 2013). The frequency of the branch modes corresponds well to the in vivo situation, with the lung model favoring lateral branching and bifurcations and the kidney model predicting robust bifurcation and trifurcation patterns, but lateral branching to be rare (Menshykau et al., 2012; Menshykau and Iber, 2013). The FGF10/SHH/PTCH-based model further predicts that the domain's growth speed influences branch mode selection, with faster growth leading to lateral branching events (Menshykau et al., 2012).

Given the dynamic nature of branching morphogenesis, a mechanistic model would additionally need to support branch outgrowth at the predicted branch points. Using timelapse movies of lung and kidney $2 \mathrm{D}$ explant cultures and developmental sequences of 3D lung buds, our group has shown that only the ligand-receptor-based Turing model correctly reproduces the areas of branch outgrowth (Menshykau et al., 2014, 2019). Tissue-restricted expression of ligands and their receptors, as observed in vivo (Figures 1A,B), makes the Turing model robust to noisy initial conditions, resulting in a geometry effect that can explain the stereotyped branching observed in the lung and the kidney (Metzger et al., 2008; Short et al., 2013; Menshykau et al., 2014, 2019). The ligand-receptor-based Turing mechanism predicts the highest signaling concentration at new branch points, which corresponds well to the in vivo pattern of pERK, a common readout of FGF10 and GDNF signaling (Chang et al., 2013; Ihermann-Hella et al., 2014; Conrad et al., 2021). Indeed, if components of the MAPK pathway are knocked out in the epithelium, no new branches can form beyond the main bronchi of the lung and the T-shape of the UB (Ihermann-Hella et al., 2014; Boucherat et al., 2015). Including the negative feedback from SHH/PTCH signaling in the FGF10/FGFR2b signaling-based model greatly increases the parameter space in which Turing patterns are observed, the so-called Turing space (Kurics et al., 2014; Menshykau et al., 2014). Likewise, the confinement of receptors to single cells and their tendency to form larger clusters greatly increases the Turing space (Kurics et al., 2014). A wider range of possible parameters allows for more variation in branching patterns and would make the evolution of a Turing mechanism more likely. Expanding the GDNF/RET-based Turing model with a positive feedback on Gdnf expression via WNT11 signaling improves the model fit to the experimental growth fields at later stages of kidney development when branch tips start to grow toward each other and compete for ligand (Menshykau et al., 2019). As predicted by the Turing mechanism, branches remain further apart in Wnt11 null kidneys (Menshykau et al., 2019). Taken together, the ligand-receptor interactions likely represent the core patterning module that drives stereotypic branching morphogenesis. Coupled with other Turing modules and further feedbacks, a wide range of patterns can be robustly achieved to enable the different stereotypic branching patterns in the different organs.

The distance at which a new branch point is specified relative to other, already formed tips has an additional impact on the shape of the branched tree. A key prediction of the ligandreceptor-based Turing mechanism is that the spacing of branch tips depends on the rate of ligand expression and is only affected if this rate falls below a threshold (Celliere et al., 2012). Wider spacing of the first three lateral branches of the left lung lobe at E12.0 is observed in Fgf10 hypomorphic lungs, but other allelic combinations with a milder reduction in Fgf10 expression did not differ from wild type lungs (Ramasamy et al., 2007). A 55\% reduction of Fgf10 expression in hypomorphic lungs compared to the wild type is in line with the simulated threshold of a $50 \%$ decrease in expression rate (Figure 2C) (Ramasamy et al., 2007; Celliere et al., 2012).

There is ample experimental evidence that biomechanical forces affect branching morphogenesis. Thus, alteration of the transmural pressure using microfluidic chambers or tracheal occlusion influences branching morphogenesis and maturation of embryonic lungs, with increased pressure leading to an increase in the formation of branches (Unbekandt et al., 2008; Nelson et al., 2017). Tracheal occlusion did not affect the final organ size, whereas the distance between branches was reduced at the culture endpoint (Unbekandt et al., 2008). Notably, the expression of Fgf10 is increased by roughly 50\%, linking the impact of the intra-luminal pressure to signaling and the Turing mechanism (Celliere et al., 2012). The changes in branch distance observed in these studies were inferred from static imaging data and do not provide information for the distance between branch 
points at the time of branch point specification. Live-imaging at high temporal resolution would circumvent this problem.

\section{Dynamic Cell Behaviors During Bud Formation}

While the genetic regulation of branch patterning is increasingly well understood, it is less clear how signaling translates to dynamic cell behaviors and which morphological processes are necessary for the formation and outgrowth of a new bud. While differential proliferation has been a longstanding candidate, there are conflicting results on its role as a driver of bud outgrowth and branching (Ettensohn, 1985). Increased proliferation in branch tips has been observed for the kidney (Michael and Davies, 2004; Riccio et al., 2016) and in distal versus proximal regions of the lung epithelium (Okubo et al., 2005). Proliferation was also shown to be restricted to already formed branch tips of mesenchyme-free lung epithelium cultures; however, proliferation was uniform before branching, arguing against differential proliferation driving bud formation in vitro (Nogawa et al., 1998).

In the lung, differential proliferation between tip and stalk was only observed for lateral branching but not during tip bifurcation (Schnatwinkel and Niswander, 2013). However, blocking proliferation in the chicken lung, which forms new branches in a comparable fashion to mouse lateral branching, showed that proliferation is not required for the initiation of new buds (Kim et al., 2013). Furthermore, computational modeling demonstrated that proliferation alone does not support lung domain branching (Kim et al., 2013; Fumoto et al., 2017; Goodwin et al., 2019). Growth-induced mechanical instability could also result in the formation of new buds through epithelial buckling (Varner et al., 2015). In this model, differential proliferation in the epithelium and the surrounding mesenchyme (or matrigel) could support bud formation, yet spatial proliferation differences within a layer are not required.

Proliferation is required for tip enlargement into the characteristic ampulla in the kidney (Michael and Davies, 2004; Ihermann-Hella et al., 2014). Nonetheless, no difference in proliferation between the bifurcating tip's lateral sides and the cleft has been observed in either the lung or the kidney, making it difficult to explain how increased tip proliferation alone could lead to bifurcations (Michael and Davies, 2004; Schnatwinkel and Niswander, 2013). Bifurcating tips in the lung showed differential cell division orientation at the newly forming tip versus the cleft region, with more divisions contributing to elongation in the cleft, potentially "pushing" the daughter tips apart (Schnatwinkel and Niswander, 2013). Luminal mitosis has been observed in the UB, during which one daughter cell loses its basal contact and reinserts into the UB a few cell diameters away (Packard et al., 2013), but it has not been demonstrated whether this process is needed for tip bifurcations and reinsertion seemingly happens in random locations.

Live-imaging of mosaic UBs has revealed a tip progenitor population that is dependent on Ret and the transcription factors Etv4/5 (Shakya et al., 2005b; Kuure et al., 2010). Tip cells compete for the tip domain based on the level of signaling and show high cell motility (Chi et al., 2009), but whether cells exhibit coordinated movement or directional migration that contributes to tip bifurcation remains to be shown. Genes downstream of Etv4/5 are involved in cellular migration, adhesion, and ECM remodeling, which could, in principle, drive cell sorting mechanisms ( $\mathrm{Lu}$ et al., 2009). GDNF has been shown to act as a chemotactic factor for the renal Madin-Darby canine kidney (MDCK) cell line. However, it is not required as a paracrine factor for UB patterning during kidney development through a localized source of GDNF (Tang et al., 1998; Shakya et al., 2005a). Similar to Ret dependent cell sorting, $F g f r 2^{U B-/-}$ cells show less frequent occupation of UB tips as compared to wildtype cells, which might provide some redundancy of GDNF and FGF signaling for cell rearrangements in the UB (Leclerc and Costantini, 2016). The matrix metalloproteinases (MMPs) MMP2 and MMP14 are localized at UB tips (Kanwar et al., 1999; Pohl et al., 2000; Meyer et al., 2004), and reduced mechanical resistance and signaling factors released by degradation of the ECM could facilitate tip outgrowth into the mesenchyme. Altering MMP activity in the lung can lead to decreased, as well as increased branching, presumably depending on the extent to which the ECM is altered (Gill et al., 2003; Rutledge et al., 2019). For example, a low concentration of an MMP inhibitor enhances focal ECM deposits, which leads to increased branching, whereas high concentrations reduce branching (Gill et al., 2006).

Ectopic basolateral localization of E-cadherin in Mek1/2 knockout $\mathrm{UB}$ and increased E-cadherin expression as a consequence of reduced FGF10 signaling in the lung suggests that increased cellular adhesion has an adverse effect on bud formation (Ihermann-Hella et al., 2014; Jones et al., 2019). Additionally, local fibronectin accumulation reduces E-cadherin in the apical cell-cell adhesion belt and fibronectin has been reported to support branching, suggesting that localized reduction of cellular adhesion allows epithelial remodeling processes that support bud bifurcation (Sakai et al., 2003). Clefting is particularly well studied in the salivary gland, and temporal imaging of fibronectin deposition in the progressing cleft has been shown to mediate bifurcation of branch tips (Larsen et al., 2006). Localized differentiation of mesenchymal-derived smooth muscle (SM) at the branch tip prior to cleft formation has been suggested to drive clefting of lung branches (Kim et al., 2015), but genetic inhibition of SM differentiation does not abrogate lung branching (Young et al., 2020).

Additionally, dynamic cell shape changes are observed during bud formation, with cells in the tips adopting a wedge-shaped morphology (Meyer et al., 2004; Kim et al., 2013; Kadzik et al., 2014). This could be achieved actively through apical constriction or be a consequence of the bud's geometry. Although this question has mainly been investigated in explant cultures supplemented with inhibitors of actomyosin contractility, studies have yielded conflicting results on the role of apical constriction (Michael et al., 2005; Moore et al., 2005; Kim et al., 2013; Schnatwinkel and Niswander, 2013; Meyer et al., 2006). Interestingly, there seems to be a requirement for apical constriction for tip bifurcation, but not for lateral branching in the mouse lung (Schnatwinkel and Niswander, 2013), while apical constriction is required for the initiation of new buds in the chicken lung (Kim et al., 2013). 


\section{Branch Orientation}

\section{Stereotyped Orientation of Branching Modes}

The first comprehensive analysis of branch angles in lungs and kidneys is provided by the morphometric studies of E14.5 lung and E15.5 ureteric trees by Short et al. (2013, 2014). Branching events in the lung occur by either domain branching or bifurcations (Metzger et al., 2008). While lateral branches in the E14.5 lung tree are oriented parallel at an angle of around $80^{\circ}$, bifurcations show a divergence angle of around $115^{\circ}$ (Figure 2A; Short et al., 2013). In the kidney, bifurcations constitute the dominant branching mode. The corresponding divergence angles in the E15.5 ureteric tree are reported to be around $100^{\circ}$ (Figure 2A; Short et al., 2013, 2014). Bifurcations do not exclusively occur in the same plane but can also be rotated relative to the previous bifurcation. In both organs, such rotations of bifurcation events show a dihedral angle of around 60-65 (Figure 2A). Therefore, branch angles for bifurcations and rotations are remarkably similar between embryonic lungs and kidneys (Short et al., 2013, 2014). In the kidney, rotational angles are constant across branch generations and developmental stages between E12.0 and E16.5 (Short et al., 2013, 2014). Overall, not only branch position but also branch orientation appears to be stereotypic in lungs and kidneys. Since the morphometric studies by Short et al. $(2013,2014)$ are endpoint analyses at certain developmental stages, they do not provide information on branch orientation at the time point of branching.

\section{Remodeling Processes and Regulation of Branch Orientation}

Branch orientation does not seem to be rigidly fixed but subject to spatial and temporal remodeling processes. Between E12.0 and E16.5, local bifurcation angles (starting direction of daughter branches) are constant across branch generations and developmental stages in the kidney, while global bifurcation angles (direction relative to terminal branch point) show substantial spatial and temporal dynamics (Short et al., 2013, 2014). The divergence angle of ureteric tips is close to $180^{\circ}$, which corresponds to the characteristic T-shape, but reduces to around $120^{\circ}$, producing a Y-shape, when the tips mature into branches (Sims-Lucas et al., 2009). While the kidney generally shows uniform growth between E12.0 and E16.5, ureteric tree and organ volume are static between E13.25 and E13.75 despite increasing tip number and density (Short et al., 2014). At around this time, compressive remodeling of the internal branches leads to an increased curvature of these internal branches in kidneys (Figure 2D; Short et al., 2013, 2014). Concurrently, terminal branches move closer toward each other (Short et al., 2013, 2014). Branch tip packaging within the organ increases with developmental time and is accompanied by a concurrent decrease of inter-tip distances (Short et al., 2010). In the kidney, the density of tips at the organ surface correlates with branching angles, as ureteric trees from $T g f-\beta 2^{+/-}$mice show increased tip distances as well as increased bifurcation angles between branching events compared to wildtypes (Short et al., 2010, 2013). Treatment of embryonic kidney cultures with TGF- $\beta 1$ significantly alters branching angles (Bush et al., 2004). Furthermore, it has been shown that branch angles are controlled by the presence of other nearby branch tips in embryonic kidney cultures (Davies et al., 2014). Interestingly, branches never collide with each other and rather avoid close contact (Miura and Shiota, 2002; Blanc et al., 2012). The ligand-receptor based Turing model explains the observed distance between ureteric tips with a competition of the opposing tips for GDNF. GDNF sets the rate of tip outgrowth, and depletion of GDNF thus stalls further outgrowth. The computational model predicts and experiments confirm that the kidney-specific positive feedback of WNT11 on GDNF enables a denser packing of ureteric branch tips (Menshykau et al., 2019). BMP7 has been suggested to negatively affect the positive feedback of WNT11 on GDNF (Goncalves and Zeller, 2011), and in vitro experiments indeed show that inhibition of BMP7 leads to branch collisions as well as small divergence angles between branches, and branches bend away from BMP7-soaked beads (Davies et al., 2014).

While the first generations of lung branching are highly stereotyped, the subsequent branching events show substantially more variations, also in the orientation of branches (Metzger et al., 2008; Blanc et al., 2012; Short et al., 2013). These variations are mainly observed in regions where spatial restrictions are less stringent, like wide-opened mesenchymal areas, suggesting that new branches grow homogeneously into the mesenchyme by following the main direction of mesenchyme growth and adapt their orientation to local changes in the mesenchyme shape (Blanc et al., 2012). How lung branches sense the mesenchymal growth pattern is still elusive. FGF10 has been proposed to act as chemoattractants in the lung since epithelial buds grow toward localized sources of FGF10 (Bellusci et al., 1997; Park et al., 1998). In vitro, lung endoderm extends toward FGF10-soaked beads independent of its association with mesenchyme (Park et al., 1998; Weaver et al., 2000). Consequently, the spatiotemporally dynamic expression pattern of Fgf10 in the lung mesenchyme has been suggested to guide directional branch outgrowth during lung branching morphogenesis (Bellusci et al., 1997; Park et al., 1998). In addition, blood vessels have been reported to direct branch orientation in the lung as vascular ablation leads to an incomplete rotation of dorsal-ventral branches (Lazarus et al., 2011). Since vascular ablation results in alterations of the spatial expression patterns of Fgf10, Shh, and Sprouty2, blood vessels may direct branch outgrowth by modulating the core signaling networks.

\section{Branch Shape and Elongation}

\section{Spatial and Temporal Dynamics of Branch Shape}

The length and width of branches in lungs and kidneys show substantial spatial and temporal dynamics during branching morphogenesis. In E15.5 kidneys, branch lengths and diameters decrease with increasing branch generation (Short et al., 2013). Similarly, E14.5 lungs present decreasing branch diameters with successive branch generation (Short et al., 2013). This trend is also observed when embryonic lung and kidney explants are cultured on a filter system (Conrad et al., 2021). Therefore, reducing branch diameters with progressive branch generations is likely a common characteristic of organ architecture in lungs 
and kidneys. Between E12.0 and E16.5, most branches of the ureteric tree increase in length and diameter over time, but not necessarily in a uniform way (Short et al., 2014). When ureteric tree and organ volume are static between E13.25 and E13.75, branching continues and branch tip length and volume but not diameter are reduced (Short et al., 2014). A multitude of studies have queried perturbations on branch shape in both organs, but it is still unknown how these complex remodeling processes are regulated in detail. Interestingly, the shape of branch stalks and tips seem to be modulated differently (Figure 2E).

\section{Biased Cell Division Accompanies Biased Branch Elongation}

The branches in both organs show anisotropic growth due to a biased mitosis spindle orientation. In the kidney, planar cell polarity (PCP) signaling is important for directing the orientation of cell divisions and tubule elongation (Saburi et al., 2008; Karner et al., 2009). For the lung, diverging reports exist on the role of the PCP pathway. Mutations in the PCP genes cadherin EGF $L A G$ seven-pass G-type receptor 1 (Celsr1) and van gogh-like2 (Vangl2) result in a reduced number of epithelial buds, but larger branch widths in embryonic lung cultures, while FGF10 signaling and cell proliferation seem to be unaffected in mutant lungs (Yates et al., 2010). In contrast to that, Tang et al., did not find any differences in branch shape or spindle orientation in lungs homozygous for Vangl2 (Tang et al., 2011). The authors rather show that the control of mitotic spindle orientation is linked to ERK1/2 signaling in the lung and that the bias in elongation is lost in mutants with a constitutively active form of Kirsten rat sarcoma viral oncogene (KRas) (Tang et al., 2011). However, independent of the involved pathway, the question arises where biased elongation originates from.

\section{Impact of Localized Signaling on Branch Shape and Elongation}

In lungs and kidneys, FGF10 and GDNF signaling is restricted to branch tips and necessary for branch formation (Moore et al., 1996; Pichel et al., 1996; Sánchez et al., 1996; Min et al., 1998; Sekine et al., 1999; Rozen et al., 2009; Michos et al., 2010; Conrad et al., 2021). However, localized signaling is not required for biased elongation since pharmacological inhibition of FGFR signaling as well as inactivation of Fgf10 or Fgfr2 does not abrogate branch elongation in embryonic lungs (Abler et al., 2009; Conrad et al., 2021). Also, in the kidney, branch initiation and elongation seem to be regulated separately since trunks have been observed to transiently elongate without the contribution of tip-derived cells (Shakya et al., 2005b), and deletion of Mek1/2 or pharmacological inhibition of MEK1 abrogates budding but not elongation (Fisher et al., 2001; Watanabe and Costantini, 2004; Ihermann-Hella et al., 2014). Similarly, hepatocyte growth factor (HGF) and TGF- $\beta$ promote elongation at the expense of branching in embryonic UB cultures (Davies et al., 1995; Ritvos et al., 1995; Bush et al., 2004). At the same time, treatment of embryonic kidney cultures with TGF- $\beta 1$ results in thicker UB stalks (Bush et al., 2004). Interestingly, inhibition of FGFR signaling or the PI3K pathway in embryonic lung cultures as well as inactivation of Fgfr2 in the kidney leads to reduced branch widths, whereas the elongation bias of the branches is not affected (Zhao et al., 2004; Wang et al., 2005; Sims-Lucas et al., 2009; Conrad et al., 2021).

\section{Impact of the Mesenchyme and ECM on Biased Outgrowth}

In the lung, SM wrapping around the epithelium starts to appear at around E11.5 and has been suggested to shape branches (Goodwin et al., 2019). However, Myocardin inactivation, which inhibits SM differentiation, has been shown to have no effect on branching morphogenesis in E14.5 lungs (Young et al., 2020). In the kidney, SM is only present at the ureter but not at the branches (Bush et al., 2006). Therefore, branch morphology in the lung and kidney cannot be controlled by SM. As lung and kidney epithelial branches elongate even in the absence of mesenchyme, both a force or a chemical source from the mesenchyme can be ruled out as general drivers of biased branch elongation (Nogawa and Ito, 1995; Qiao et al., 1999; Varner et al., 2015; Conrad et al., 2021). Notably, UB branches elongate and thin less in the absence of mesenchyme, suggesting that the mesenchyme affects but is not required for biased elongation (Conrad et al., 2021).

The ECM is generated both by epithelial and mesenchymal cells and thus exists also in mesenchyme-free cultures. The ECM is mainly composed of laminin, collagen, and fibronectin and is extensively remodeled during branching morphogenesis (Harunaga et al., 2015; Kyprianou et al., 2020). MMPs degrade ECM components and are therefore important regulators of ECM thickness. Since the ECM is thinner at branch tips, it facilitates the invasion of epithelial buds into the mesenchyme, suggesting that ECM remodeling might drive biased branch elongation. Deletion of Adamts18, an MMP-encoding gene with branch-tip enriched expression, leads to distinct phenotypes in lungs and kidneys (Rutledge et al., 2019). While Adamts18-/lungs show reduced branching and shorter primary airways, Adamts $18^{-/-}$kidneys develop two ureters rather than one, while branching seems to be unaffected. Adamts18 knockout embryos do not show any difference in Fgf10, Shh, Bmp4, and Sprouty2 expression. In contrast to Adamts18 null lungs, Adamts $18^{+/-}$ lungs exhibit increased branch formation, suggesting a complex relationship between MMP levels and branching. UBs secrete endostatin (ES), a cleavage product of collagen XVIII, by MMPdriven degradation and bind ES along the stalks, but not at the branch tips. Interestingly, the presence of recombinant murine ES inhibits outgrowth and branching of embryonic rat UB cultured in Matrigel, resulting in short and widened branches. The presence of an ES-neutralizing antibody enhances UB outgrowth and branching, resulting in increased branch lengths (Karihaloo et al., 2001). However, despite the impact of these local ECM modulations on branch growth and shape, pharmacological inhibition of MMPs does not affect the elongation bias in embryonic lungs (Conrad et al., 2021).

\section{Cell-Based Simulations Suggest a Pulling Rather Than a Constricting Force to Drive Branch Outgrowth} As we have seen before, the mesenchyme and ECM are unlikely to drive the elongation of epithelial branches by the action of compressive forces. Moreover, cell-based tissue simulations of 
epithelial lung growth demonstrate that external constricting forces that lead to the bias in outgrowth observed in embryonic lung epithelium, do not yield the observed bias in cell shape and division (Stopka et al., 2019). Therefore, another mechanism has to be at play to generate the bias in outgrowth. Further cell-based tissue simulations revealed that a pulling force in longitudinal direction reproduces the observed bias in outgrowth as well as in cell shape and division (Conrad et al., 2021). Actin-rich protrusions at branch tips could, in principle, generate such a pulling force that drives biased elongation but are not observed at branch tips in lungs (Conrad et al., 2021).

\section{Fluid Flow as a Driver of Biased Branch Outgrowth}

Branches in embryonic lungs and kidneys show largely collapsed tubular morphologies with narrow luminal spaces (Conrad et al., 2021). Simulations of tube collapse show that mechanical deformations would result in non-uniform stress and curvature patterns in the tube cross-section which would be inconsistent with uniform biased outgrowth (Conrad et al., 2021). Accordingly, actin density, which has been reported to be affected by external mechanical stimuli, is distributed relatively uniformly in collapsed lung branches (Hirata et al., 2008; Hayakawa et al., 2011; Shao et al., 2015; Conrad et al., 2021).

Recently, fluid flow inside branches has been quantitatively analysed during early lung development (Conrad et al., 2021). Flow-induced shear stress could act as a tangential force on the apical side of epithelial cells, which in turn, could drive elongating outgrowth. Cells are not directly deformed by shear stress but sense the stress via primary cilia and respond by adapting their cell shape (Galbraith et al., 1998; Jain et al., 2010; Weinbaum et al., 2011). For the narrow luminal spaces of the collapsed tubes and the estimated flow velocity, the estimated shear stress levels are well within the range that has been reported for cells to sense via primary cilia (Resnick and Hopfer, 2007; Flitney et al., 2009; Nauli et al., 2013). Therefore, shear stress acting in the longitudinal direction on the apical side of branches is able to explain biased elongation as well as cell division and may play an essential role in driving elongating outgrowth in lungs and kidneys (Conrad et al., 2021).

\section{Branch Tip Shape \\ Regulation of Branch Tip Shape by Core Signaling Networks}

Besides the shape and elongation of branch stalks, the bud tips are highly sensitive to pharmacological treatments and mutations. FGF10 and GDNF signaling are essential for growth and branching in lung and kidney, respectively, but are linked to branch shape regulation as well. FGF7, an alternative FGFR2b ligand, inhibits branching and results in dilated bud phenotypes in lung cultures (Simonet et al., 1995; Cardoso et al., 1997; Park et al., 1998; Tichelaar et al., 2000). While FGF10 triggers receptor recycling and cell migration, FGF7 induces receptor degradation and cell proliferation (Francavilla et al., 2013). FGF9 treatment leads to lung bud dilation and up-regulation of Fgf10 expression in the mesenchyme (del Moral et al., 2006; White et al., 2006; Yin et al., 2011; Yin and Ornitz, 2020). The effect of FGF9 on lung buds is observed even if Mek is deleted, suggesting that FGF9 mediates its effect via MEK-independent signaling pathways (Boucherat et al., 2015). It has been recently demonstrated that FGF9 signals via FGFR3, activates PI3K and thereby promotes distal epithelial fate specification and inhibits epithelial differentiation in the lung (Yin and Ornitz, 2020). Hyperactive Kras and pharmacological inhibition of MEK, both downstream of FGFR2 signaling, induce dilated branch tips in the lung (Fisher et al., 2001; Chang et al., 2013; Boucherat et al., 2015; Yin and Ornitz, 2020). In the lung, FGF10 is engaged in a negative feedback with $\mathrm{SHH}$, meaning that FGF10 signaling induces Shh expression, which in turn represses Fgf10 expression (Figure 1C; Bellusci et al., 1997; Lebeche et al., 1999). Inhibition of $\mathrm{SHH}$ signaling by cyclopamine results in an increased number of small distal buds in lung cultures, while the stalks show an inflated phenotype (White et al., 2006).

In kidney cultures, adding GDNF either uniformly or loaded on beads leads to bud widening (Pepicelli et al., 1997; Sainio et al., 1997; Shakya et al., 2005a; Menshykau et al., 2019). Dilated tips are also observed in UBs in which increased Gdnf expression is restricted to cells that naturally transcribe $G d n f$ ( $\mathrm{Li}$ et al., 2019). This phenotype is reversed by pharmacological MEK inhibition and is suggested to be a result of restricted emigration of cells from tip to stalk regions and shortened cell-cycle time in tip cells (Li et al., 2019). Deletion of the negative regulator Sprouty1 increases branching and Gdnf expression and results in swollen UB tips at the same time (Basson et al., 2005, 2006; Michos et al., 2010). In double knockout mice for Gdnf and Sprouty1, FGF10 signaling is sufficient for UB branching but some UB tips are irregularly shaped (Michos et al., 2010). Loss of phosphatase and tensin homolog (PTEN), which antagonizes the PI3K signaling pathway, leads to dilated UB tips, strongly resembling the bud morphology reported for GDNF excess or expression of a constitutively active form of RET (Pepicelli et al., 1997; Sainio et al., 1997; de Graaff, 2001; Kim and Dressler, 2007; Menshykau et al., 2019).

\section{Regulation of Branch Tip Shape by ECM Remodeling}

The ECM has been shown to influence branch morphology in lungs, kidneys and salivary glands. Treatment with an antilaminin antibody or collagenase reduces lung branching and leads to dilatation of branches (Ganser et al., 1991; Schuger et al., 1991; Miura and Shiota, 2002). Collagenase treatment results in a dilated phenotype in the kidney and salivary gland, but not in the pancreas (Wessells and Cohen, 1968). In the lung, dysregulation of Sox9 expression induces cyst-like structures at branch tips and defects in laminin and collagen disposition (Chang et al., 2013; Rockich et al., 2013). In the lung, kidney, and salivary gland, fibronectin inhibition blocks cleft formation and branching, resulting in enlarged buds, while supplementation with exogenous fibronectin enhances branching morphogenesis (Sakai et al., 2003; De Langhe et al., 2005). Notably, accumulation of fibronectin in cleft regions is accompanied by an adjacent loss of E-cadherin localization, suggesting that fibronectin might regulate branching morphogenesis by converting cell-cell adhesion into cell-matrix adhesions (Sakai et al., 2003; Onodera et al., 2010). Moreover, fibronectin is suggested to be involved in WNT-regulated morphogenetic processes as inhibition of 
WNT signaling in the lung by Dickkopf-1 (DKK1) treatment results in decreased fibronectin deposition, impaired branching, dilated end buds, decreased SM actin expression and defects in vasculature formation (De Langhe et al., 2005). The expression of Fgf10, Bmp4, and Shh is unaffected upon DKK1 treatment, indicating that the observed dilated branch shape is not due to the altered expression of these key regulatory molecules in lung branching morphogenesis. Notably, FGF9, which induces bud dilation in lung cultures, has been reported to increase $D k k 1$ expression (del Moral et al., 2006).

During branching morphogenesis, the ECM is extensively remodeled by the degrading action of MMPs (Harunaga et al., 2015; Kyprianou et al., 2020). Null mutation for tissue inhibitor of metalloproteinases 3 (TIMP-3) leads to increased activation of MMPs, enhanced fibronectin degradation and dilated buds in lungs (Gill et al., 2003, 2006). Similarly, treatment of embryonic lungs with an MMP inhibitor at high concentrations impairs branching and induces the dilation of tips (Gill et al., 2003, 2006). Interestingly, low concentrations of the MMP inhibitor result in enhanced growth and branching (Gill et al., 2003, 2006). Epidermal growth factor (EGF), TGF- $\alpha$ and FGF7 induce dilation of branch tips and are suggested to induce MMP activity in the lung (Ganser et al., 1991; Miura and Shiota, 2002).

Moreover, efficient RTK signaling of FGF10 and GDNF requires sulphated glycosaminoglycans (GAG) that are expressed in the ECM. Inhibition of GAG synthesis by sodium chlorate disrupts branching morphogenesis in lungs and kidneys (Davies et al., 1995; Kispert et al., 1996; Shannon et al., 2003; Michael et al., 2005). In the lung, heparan sulfate proteoglycans are important for FGF10 binding to the distal epithelium and treatment with heparinase or oversulphated heparins leads to dilated lung buds (Izvolsky et al., 2003).

\section{Regulation of Branch Tip Shape by Cytoskeleton Dynamics}

Epithelial cell shape and tension dynamics mediated by the actinmyosin cytoskeleton have been shown to play an important role during branching morphogenesis (Moore et al., 2005; Kadzik et al., 2014). In the kidney, chemical inhibition of myosin ATPase, disruption of actin microfilaments, or inhibition of rhoassociated protein kinase (ROCK) inhibits branching and results in bloated and misshapen bud tips (Michael et al., 2005). While chemical inhibition of myosin ATPase or disruption of actin microfilament integrity block growth and branching in the lung, but do not seem to affect tip morphology, suppression of myosin light chain (MLC) phosphorylation by pharmacological ROCK or MLC kinase inhibition leads to a lung phenotype with dilated buds (Moore et al., 2005; Kadzik et al., 2014). In the lung, ROCK inhibition disrupts differential growth patterns, actin and ECM remodeling as well as the organization of vascular architecture and epithelial morphology (Moore et al., 2005). Wnt/Frizzled2 (Fzd2) signaling is required for controlling cell shape changes by regulating ras homolog family member A (RhoA), which in turn activates ROCK. Deletion of the Wnt receptor $F z d 2$ results in decreased RhoA activity, decreased phosphorylated MLC levels at apical cell surfaces as well as dilated distal buds in lungs (Kadzik et al., 2014). Notably, these Fzd2 lung mutants show elevated and expanded Fgf10 expression, which potentially contributes to the overall phenotype, while the expression patterns of $\mathrm{Fg} f \mathrm{f} 2 \mathrm{~b}, \mathrm{Sh}$, and $B m p 4$ are not altered.

\section{Regulation of Branch Tip Shape by Cellular Transport Components}

Epithelia of developing lungs, kidneys, and salivary glands express several types of voltage-dependent calcium channels (VDCCs) that have been linked to branching and branch shape. Inhibition of VDCCs by nifedipine treatment leads to abrogated branching, dilated buds, and reduced ERK activation, suggesting a link between VDCC activity and ERK phosphorylation (Kim et al., 2015, 2018). In the kidney, removal of a subset of claudins, essential components of tight junctions and important for paracellular transport, leads to a decrease in ureteric tip formation, enlarged bud tip lumens and a decreased complexity regarding tip morphology (El Andalousi et al., 2020).

\section{Regulation of Branch Tip Shape by Vasculature}

Interestingly, blood vessels have been shown to influence branch shape in the lung. Disruption of vascular assembly by overexpression of vascular endothelial growth factor-A (VEGFA) decreases branching and dilates branch tips in the lung (Akeson et al., 2003). Similarly, vascular inhibition leads to dilated airway branches and reduced branching, while cell proliferation remains unaffected (Lazarus et al., 2011). Notably, vascular ablation alters the expression pattern of Fgf10 and the expression levels of Shh and Sprouty2. The resulting perturbed branching phenotype resembles the phenotype caused by ectopic Fgf10 expression.

\section{Regulation of Branch Tip Shape by RNA Molecules}

Finally, RNA molecules have been reported to affect branching morphogenesis (Yu, 2014). Two microRNAs (miRNAs) that have originally been documented to regulate angiogenesis, the proangiogenic miR-221, and the anti-angiogenic miR-130, show similar opposing effects on branch tip morphology in the lung (Mujahid et al., 2013). While downregulation of miR221 enhances branching and vascular network formation but decreases tip width, anti-miR-130 treatment results in reduced branching, a poorly developed vascular network, and dilated branch tips (Mujahid et al., 2013). Accordingly, upregulation of these miRNAs triggers the corresponding inverse effect on lung branching, vascular network formation and tip morphology (Mujahid et al., 2013). Notably, one target of miR-221 is the Hox gene Hoxb5 which, in turn, has been reported to affect the expression of tenascin- $C$ in the ECM as well as the spatial restriction of Fgf10 expression in the lung mesenchyme (Volpe et al., 2007).

Dicer is an endoribonuclease that is expressed in the lung at the onset of branching morphogenesis and processes miRNA and small interfering RNA into their mature forms, which in turn regulate gene expression. Epithelial inactivation of Dicer results in the arrest of new branch formation and the dilation of distal tips, while epithelial growth is unaffected (Harris et al., 2006). Moreover, Dicer null-mutants show increased Fgf10, Sprouty2, and Bmp4 expression. In the kidney, epithelial removal of Dicer 
leads to the formation of dilated cysts, disrupted branching morphogenesis, and the downregulation of Wnt11 and Ret, while Gdnf expression is unaffected (Nagalakshmi et al., 2011).

\section{CONCLUSION AND OUTLOOK}

In this review, we compared branching morphogenesis in lungs and kidneys on the branch-level by focusing on developmental mechanisms that determine branch position, orientation, and shape. In both organs, epithelial-mesenchymal signaling interactions, but also the ECM, and the cytoskeleton appear to play crucial roles in regulating these hallmarks of branching. However, the presented findings are primarily based on the analysis of individual snapshots during development or qualitative descriptions of perturbed branching morphogenesis, meaning that data on branching characteristics at the time point of branch formation is mostly missing. As such, quantitative time-lapse analyses of branch position, orientation, and shape during lung and kidney development are needed to gain further insights into the regulation of organ-specific branching morphogenesis.

Tissue recombination experiments have demonstrated the mesenchyme's ability to establish organ-specific branching patterns (Lin et al., 2003). It is not unlikely that the mesenchyme modulates additional determinants of the branched tree, like the angles between parent and daughter branches and the final branch shapes, although these aspects have not been quantified in tissue recombination experiments. Comprehensive analyses of the impact of the mesenchyme on branching hallmarks are

\section{REFERENCES}

Abler, L. L., Mansour, S. L., and Sun, X. (2009). Conditional gene inactivation reveals roles for Fgf10 and Fgfr2 in establishing a normal pattern of epithelial branching in the mouse lung. Dev. Dyn. 238, 1999-2013. doi: 10.1002/dvdy. 22032

Affolter, M., and Caussinus, E. (2008). Tracheal branching morphogenesis in Drosophila: new insights into cell behaviour and organ architecture. Development 135, 2055-2064. doi: 10.1242/dev.014498

Akeson, A. L., Greenberg, J. M., Cameron, J. E., Thompson, F. Y., Brooks, S. K., Wiginton, D., et al. (2003). Temporal and spatial regulation of VEGF-A controls vascular patterning in the embryonic lung. Dev. Biol. 264, 443-455. doi: 10. 1016/j.ydbio.2003.09.004

Alescio, T., and Dani, A. M. (1971). The influence of mesenchyme on the epithelial glycogen and budding activity in mouse embryonic lung developing in vitro. J. Embryol. Exp. Morph. Vol 25, 131-140. doi: 10.1242/dev.25.1.131

Basson, M. A., Akbulut, S., Watson-Johnson, J., Simon, R., Carroll, T. J., Shakya, R., et al. (2005). Sproutyl is a critical regulator of GDNF/RET-mediated kidney induction. Dev. Cell 8, 229-239. doi: 10.1016/j.devcel.2004.12.004

Basson, M. A., Watson-Johnson, J., Shakya, R., Akbulut, S., Hyink, D., Costantini, F. D., et al. (2006). Branching morphogenesis of the ureteric epithelium during kidney development is coordinated by the opposing functions of GDNF and sprouty. Dev. Biol. 12, 466-477. doi: 10.1016/j.ydbio.2006.08.051

Bellusci, S., Grindley, J., Emoto, H., Itoh, N., and Hogan, B. L. M. (1997). Fibroblast Growth Factor 10 (FGF10) and branching morphogenesis in the embryonic mouse lung. Development 124, 4867-4878. doi: 10.1242/dev.124.23.4867

Blanc, P., Coste, K., Pouchin, P., Azaïs, J.-M., Blanchon, L., Gallot, D., et al. (2012). A role for mesenchyme dynamics in mouse lung branching morphogenesis. PLoS One 7:e41643. doi: 10.1371/journal.pone.0041643 thus required to elucidate further the role of the mesenchyme in shaping epithelial trees in different organs.

Besides establishing a highly branched epithelial tree, other developmental processes are equally essential for shaping organs. Already during branching morphogenesis, the functional units start to form, i.e., the acini in the lung and the nephrons in the kidney (McMahon, 2016; Schittny, 2017), accompanied by highly complex remodeling processes. As a consequence, unlike the epithelial tree structure that is laid down during lung branching morphogenesis, the fully developed adult lung exhibits a fractal-like architecture (Weibel, 1991). In the kidney, nephron formation comprises remodeling processes such as the elongation and tight packaging of unbranched tubules (Little et al., 2010). Combining live imaging, quantitative image analysis, and mathematical modeling will provide essential insights into the regulation of these remodeling processes and their contribution to organ-specific development.

\section{AUTHOR CONTRIBUTIONS}

CL and LC wrote the manuscript. DI conceived and edited the manuscript. All authors contributed to the article and approved it for publication.

\section{ACKNOWLEDGMENTS}

We thank members of the CoBi group for their feedback and discussions.

Boucherat, O., Nadeau, V., Berube-Simard, F.-A., Charron, J., and Jeannotte, L. (2015). Crucial requirement of ERK/MAPK signaling in respiratory tract development. Development 142, 3801-3801. doi: 10.1242/dev.131821

Bracken, C. M., Mizeracka, K., and McLaughlin, K. A. (2008). Patterning the embryonic kidney: BMP signaling mediates the differentiation of the pronephric tubules and duct in Xenopus laevis. Dev. Dyn. 237, 132-144. doi: 10.1002/dvdy.21387

Brunskill, E. W., Park, J.-S., Chung, E., Chen, F., Magella, B., and Potter, S. S. (2014). Single cell dissection of early kidney development: multilineage priming. Development 141, 3093-3101. doi: 10.1242/dev.110601

Bush, K. T., Sakurai, H., Steer, D. L., Leonard, M. O., Sampogna, R. V., Meyer, T. N., et al. (2004). TGF-h superfamily members modulate growth, branching, shaping, and patterning of the ureteric bud. Dev. Biol. 14, 285-298. doi: 10. 1016/j.ydbio.2003.10.023

Bush, K. T., Vaughn, D. A., Li, X., Rosenfeld, M. G., Rose, D. W., Mendoza, S. A., et al. (2006). Development and differentiation of the ureteric bud into the ureter in the absence of a kidney collecting system. Dev. Biol. 298, 571-584. doi: 10.1016/j.ydbio.2006.07.006

Cacalano, G., Wang, L.-C., Hagler, K., Forgie, A., Moore, M., Armanini, M., et al. (1998). GFR $\alpha 1$ is an essential receptor component for GDNF in the developing nervous system and kidney. Neuron 21, 53-62. doi: 10.1016/s0896-6273(00) 80514-0

Cardoso, W. V., Itoh, A., Nogawa, H., Mason, I., and Brody, J. S. (1997). FGF-1 and FGF-7 induce distinct patterns of growth and differentiation in embryonic lung epithelium. Dev. Dyn. 208, 398-405. doi: 10.1002/(sici)1097-0177(199703)208: 3<398::aid-aja10>3.0.co;2-x

Cardoso, W. V., and Lü, J. (2006). Regulation of early lung morphogenesis: questions, facts and controversies. Development 133, 1611-1624. doi: 10.1242/ dev.02310 
Cebrián, C., Borodo, K., Charles, N., and Herzlinger, D. A. (2004). Morphometric index of the developing murine kidney: morphometric index of the mouse kidney. Dev. Dyn. 231, 601-608. doi: 10.1002/dvdy.20143

Celliere, G., Menshykau, D., and Iber, D. (2012). Simulations demonstrate a simple network to be sufficient to control branch point selection, smooth muscle and vasculature formation during lung branching morphogenesis. Biol. Open 1, 775-788. doi: 10.1242/bio.20121339

Chang, D. R., Alanis, D. M., Miller, R. K., Ji, H., Akiyama, H., McCrea, P. D., et al. (2013). Lung epithelial branching program antagonizes alveolar differentiation. PNAS 110, 18042-18051. doi: 10.1073/pnas.1311760110

Chi, X., Michos, O., Shakya, R., Riccio, P., Enomoto, H., Licht, J. D., et al. (2009). Ret-dependent cell rearrangements in the wolffian duct epithelium initiate ureteric bud morphogenesis. Dev. Cell 17, 199-209. doi: 10.1016/j.devcel.2009. 07.013

Conrad, L., Runser, S., Gómez, H., Lang, C., Dumond, M., Sapala, A., et al. (2021). The biomechanical basis of biased epithelial tube elongation in lung and kidney development. biorxiv [Preprint]. doi: 10.1101/2020.06.22.166231

Costantini, F., and Kopan, R. (2010). Patterning a complex organ: branching morphogenesis and nephron segmentation in kidney development. Dev. Cell 18, 698-712. doi: 10.1016/j.devcel.2010.04.008

Davies, J., Lyon, M., Gallagher, J., and Garrod, D. (1995). Sulphated proteoglycan is required for collecting duct growth and branching but not nephron formation during kidney development. Development 121, 1507-1517. doi: 10.1242/dev. 121.5.1507

Davies, J. A., Hohenstein, P., Chang, C.-H., and Berry, R. (2014). A self-avoidance mechanism in patterning of the urinary collecting duct tree. BMC Dev. Biol. 14:35. doi: 10.1186/s12861-014-0035-8

de Graaff, E. (2001). Differential activities of the RET tyrosine kinase receptor isoforms during mammalian embryogenesis. Genes Dev. 15, 2433-2444. doi: 10.1101/gad.205001

De Langhe, S. P., Sala, F. G., Del Moral, P.-M., Fairbanks, T. J., Yamada, K. M., Warburton, D., et al. (2005). Dickkopf-1 (DKK1) reveals that fibronectin is a major target of Wnt signaling in branching morphogenesis of the mouse embryonic lung. Dev. Biol. 277, 316-331. doi: 10.1016/j.ydbio.2004. 09.023

del Moral, P.-M., De Langhe, S. P., Sala, F. G., Veltmaat, J. M., Tefft, D., Wang, K., et al. (2006). Differential role of FGF9 on epithelium and mesenchyme in mouse embryonic lung. Dev. Biol. 293, 77-89. doi: 10.1016/j.ydbio.2006.01.020

del Moral, P.-M., and Warburton, D. (2010). Explant culture of mouse embryonic whole lung, isolated epithelium, or mesenchyme under chemically defined conditions as a system to evaluate the molecular mechanism of branching morphogenesis and cellular differentiation. Mouse Cell Culture 633, 71-79. doi: 10.1007/978-1-59745-019-5_5

Duernberger, H., and Kratochwil, K. (1980). Specificity of tissue interaction and origin of mesenchymal cells in the androgen response of the embryonic mammary gland. Cell 19, 465-471. doi: 10.1016/0092-8674(80)90521-8

El Andalousi, J., Khairallah, H., Zhuang, Y., Ryan, A. K., and Gupta, I. R. (2020). Role of claudins in renal branching morphogenesis. Physiol. Rep. 8:e14492.

Ettensohn, C. A. (1985). Mechanisms of epithelial invagination. Q. Rev. Biol. 60, 289-307. doi: 10.1086/414426

Fisher, C. E., Michael, L., Barnett, M. W., and Davies, J. A. (2001). Erk MAP kinase regulates branching morphogenesis in the developing mouse kidney. Development 128, 4329-4338. doi: 10.1242/dev.128.21.4329

Flitney, E. W., Kuczmarski, E. R., Adam, S. A., and Goldman, R. D. (2009). Insights into the mechanical properties of epithelial cells: the effects of shear stress on the assembly and remodeling of keratin intermediate filaments. FASEB J. 23, 2110-2119. doi: 10.1096/fj.08-124453

Francavilla, C., Rigbolt, K. T. G., Emdal, K. B., Carraro, G., Vernet, E., BekkerJensen, D. B., et al. (2013). Functional proteomics defines the molecular switch underlying FGF receptor trafficking and cellular outputs. Mol. Cell 51, 707-722. doi: 10.1016/j.molcel.2013.08.002

Fumoto, K., Takigawa-Imamura, H., Sumiyama, K., Kaneiwa, T., and Kikuchi, A. (2017). Modulation of apical constriction by Wnt signaling is required for lung epithelial shape transition. Development 144, 151-162. doi: 10.1242/dev.14 1325

Galbraith, C. G., Skalak, R., and Chien, S. (1998). Shear stress induces spatial reorganization of the endothelial cell cytoskeleton. Cell Motil. Cytoskelet. 40, 317-330. doi: 10.1002/(sici)1097-0169(1998)40:4<317::aid-cm1>3.0.co;2-8
Ganser, G. L., Stricklin', G. P., and Matrisian, L. M. (1991). EGF and TGFa influence in vitro lung development by the induction of matrix-degrading metalloproteinases. Int. J. Dev. Biol. 35, 453-461.

Gierer, A., and Meinhardt, H. (1972). A theory of biological pattern formation. Kybernetik 12, 30-39. doi: 10.1007/bf00289234

Gill, S. E., Pape, M. C., Khokha, R., Watson, A. J., and Leco, K. J. (2003). A null mutation for tissue inhibitor of metalloproteinases-3 (Timp-3) impairs murine bronchiole branching morphogenesis. Dev. Biol. 261, 313-323. doi: 10.1016/s0012-1606(03)00318-x

Gill, S. E., Pape, M. C., and Leco, K. J. (2006). Tissue inhibitor of metalloproteinases 3 regulates extracellular matrix - Cell signaling during bronchiole branching morphogenesis. Dev. Biol. 15, 540-554. doi: 10.1016/j.ydbio.2006.07.004

Goncalves, A., and Zeller, R. (2011). Genetic analysis reveals an unexpected role of BMP7 in initiation of ureteric bud outgrowth in mouse embryos. PLoS One 6:e0019370. doi: 10.1371/journal.pone.0019370

Goodwin, K., Mao, S., Guyomar, T., Miller, E., Radisky, D. C., Košmrlj, A., et al. (2019). Smooth muscle differentiation shapes domain branches during mouse lung development. Development 146:dev181172.

Goodwin, K., and Nelson, C. M. (2020). Branching morphogenesis. Development 147:dev184499.

Grobstein, C. (1955). Inductive interaction in the development of the mouse metanephros. J. Exp. Zool. 130, 319-339. doi: 10.1002/jez.1401300207

Harris, K. S., Zhang, Z., McManus, M. T., Harfe, B. D., and Sun, X. (2006). Dicer function is essential for lung epithelium morphogenesis. PNAS 103, 2208-2213. doi: 10.1073/pnas.0510839103

Harunaga, J. S., Doyle, A. D., and Yamada, K. M. (2015). Local and global dynamics of the basement membrane during branching morphogenesis require protease activity and actomyosin contractility. Dev. Biol. 394, 197-205.

Hashimoto, S., Chen, H., Que, J., Brockway, B. L., Drake, J. A., Snyder, J. C., et al. (2012). $\beta$-Catenin-SOX2 signaling regulates the fate of developing airway epithelium. J. Cell. Sci. 125, 932-942. doi: 10.1242/jcs.092734

Hayakawa, K., Tatsumi, H., and Sokabe, M. (2011). Actin filaments function as a tension sensor by tension-dependent binding of cofilin to the filament. J. Cell Biol. 195, 721-727. doi: 10.1083/jcb.201102039

Hellmich, H. L., Kos, L., Cho, E. S., Mahon, K. A., and Zimmer, A. (1996). Embryonic expression of glial cell-line derived neurotrophic factor (GDNF) suggests multiple developmental roles in neural differentiation and epithelialmesenchymal interactions. Mech. Dev. 54, 95-105. doi: 10.1016/0925-4773(95) 00464-5

Herriges, J. C., Verheyden, J. M., Zhang, Z., Sui, P., Zhang, Y., Anderson, M. J., et al. (2015). FGF-regulated ETV transcription factors control FGF-SHH feedback loop in lung branching. Dev. Cell 35, 322-332. doi: 10.1016/j.devcel.2015.10.006

Herriges, M., and Morrisey, E. E. (2014). Lung development: orchestrating the generation and regeneration of a complex organ. Development 141, 502-513. doi: $10.1242 /$ dev.098186

Hirata, H., Tatsumi, H., and Sokabe, M. (2008). Mechanical forces facilitate actin polymerization at focal adhesions in a zyxin-dependent manner. J. Cell Sci. 121, 2795-2804. doi: 10.1242/jcs.030320

Horowitz, A., and Simons, M. (2008). Branching morphogenesis. Circ. Res. 103, 784-795.

Iber, D., and Menshykau, D. (2013). The control of branching morphogenesis. Open Biol. 3:130088. doi: 10.1098/rsob.130088

Ihermann-Hella, A., Lume, M., Miinalainen, I. J., Pirttiniemi, A., and Gui, Y. (2014). Mitogen-activated protein kinase (MAPK) pathway regulates branching by remodeling epithelial cell adhesion. PLoS Genet. 10:e1004193. doi: 10.1371/ journal.pgen.1004193

Izvolsky, K. I., Zhong, L., Wei, L., Yu, Q., Nugent, M. A., and Cardoso, W. V. (2003). Heparan sulfates expressed in the distal lung are required for Fgf10 binding to the epithelium and for airway branching. Am. J. Physiol. Lung Cell. Mol. Physiol. 285:9.

Jain, R., Pan, J., Driscoll, J. A., Wisner, J. W., Huang, T., Gunsten, S. P., et al. (2010). Temporal relationship between primary and motile ciliogenesis in airway epithelial cells. Am. J. Respir. Cell Mol. Biol. 43, 731-739. doi: 10.1165/ rcmb.2009-0328oc

Jing, S., Wen, D., Yu, Y., Holst, P. L., Luo, Y., Fang, M., et al. (1996). GDNFinduced activation of the ret protein tyrosine kinase is mediated by GDNFR- $\alpha$, a novel receptor for GDNF. Cell 85, 1113-1124. doi: 10.1016/s0092-8674(00) 81311-2 
Jones, M. R., Chong, L., and Bellusci, S. (2021). Fgf10/Fgfr2b signaling orchestrates the symphony of molecular, cellular, and physical processes required for harmonious airway branching morphogenesis. Front. Cell Dev. Biol. 8:620667. doi: $10.3389 /$ fcell.2020.620667

Jones, M. R., Dilai, S., Lingampally, A., Chao, C.-M., Danopoulos, S., Carraro, G., et al. (2019). A comprehensive analysis of fibroblast growth factor receptor $2 \mathrm{~b}$ signaling on epithelial tip progenitor cells during early mouse lung branching morphogenesis. Front. Genet. 9:746. doi: 10.3389/fgene.2018.00746

Kadzik, R. S., Cohen, E. D., Morley, M. P., Stewart, K. M., Lu, M. M., and Morrisey, E. E. (2014). Wnt ligand/Frizzled 2 receptor signaling regulates tube shape and branch-point formation in the lung through control of epithelial cell shape. Proc. Natl. Acad. Sci. U.S.A. 111, 12444-12449. doi: 10.1073/pnas.1406639111

Kanwar, Y. S., Ota, K., Yang, Q., Wada, J., Kashihara, N., Tian, Y., et al. (1999). Role of membrane-type matrix metalloproteinase 1 (MT-1-MMP), MMP-2, and its inhibitor in nephrogenesis. Am. J. Physiol. Renal Physiol. 277, F934-F947.

Karihaloo, A., Karumanchi, S. A., Barasch, J., Jha, V., Nickel, C. H., Yang, J., et al. (2001). Endostatin regulates branching morphogenesis of renal epithelial cells and ureteric bud. Cell Biol. 6, 12509-12514. doi: 10.1073/pnas.221205198

Karner, C. M., Chirumamilla, R., Aoki, S., Igarashi, P., Wallingford, J. B., and Carroll, T. J. (2009). Wnt9b signaling regulates planar cell polarity and kidney tubule morphogenesis. Nat. Genet. 41, 793-799. doi: 10.1038/ng.400

Kim, D., and Dressler, G. R. (2007). PTEN modulates GDNF/RET mediated chemotaxis and branching morphogenesis in the developing kidney. Dev. Biol. 307, 290-299. doi: 10.1016/j.ydbio.2007.04.051

Kim, H. Y., Pang, M.-F., Varner, V. D., Kojima, L., Miller, E., Radisky, D. C., et al. (2015). Localized smooth muscle differentiation is essential for epithelial bifurcation during branching morphogenesis of the mammalian lung. Dev. Cell 34, 719-726. doi: 10.1016/j.devcel.2015.08.012

Kim, H. Y., Varner, V. D., and Nelson, C. M. (2013). Apical constriction initiates new bud formation during monopodial branching of the embryonic chicken lung. Development 140, 3146-3155. doi: 10.1242/dev.093682

Kim, J. M., Choi, S., Lee, S. W., and Park, K. (2018). Voltage-dependent Ca2+ channels promote branching morphogenesis of salivary glands by patterning differential growth. Sci. Rep. 11:7566.

Kispert, A., Vainio, S., Shen, L., Rowitch, D. H., and McMahon, A. P. (1996). Proteoglycans are required for maintenance of Wnt-11 expression in the ureter tips. Development 122, 3627-3637. doi: 10.1242/dev.122.11.3627

Kratochwil, K. (1969). Organ specificity in mesenchymal induction demonstrated in the embryonic development of the mammary gland of the mouse. Dev. Biol. 20, 46-71. doi: 10.1016/0012-1606(69)90004-9

Kurics, T., Menshykau, D., and Iber, D. (2014). Feedback, receptor clustering, and receptor restriction to single cells yield large Turing spaces for ligand-receptorbased turing models. Phys. Rev. E 90:022716.

Kuure, S., Chi, X., Lu, B., and Costantini, F. (2010). The transcription factors Etv4 and Etv5 mediate formation of the ureteric bud tip domain during kidney development. Development 137, 1975-1979. doi: 10.1242/dev.051656

Kyprianou, C., Christodoulou, N., Hamilton, R. S., Nahaboo, W., Boomgaard, D. S., Amadei, G., et al. (2020). Basement membrane remodelling regulates mouse embryogenesis. Nature 582, 253-258. doi: 10.1038/s41586-020-2264-2

Lang, C., Conrad, L., and Michos, O. (2018). Mathematical approaches of branching morphogenesis. Front. Genet. 9:673. doi: 10.3389/fgene.2018.00673

Larsen, M., Wei, C., and Yamada, K. M. (2006). Cell and fibronectin dynamics during branching morphogenesis. J. Cell Sci. 119(Pt 16), 3376-3384. doi: 10. $1242 /$ jcs. 03079

Lawson, K. A. (1983). Stage specificity in the mesenchyme requirement of rodent lung epithelium in vitro: a matter of growth control? J. Embryol. Exp. Morph. 74, 183-206. doi: 10.1242/dev.74.1.183

Lazarus, A., Del-Moral, P. M., Ilovich, O., Mishani, E., Warburton, D., and Keshet, E. (2011). A perfusion-independent role of blood vessels in determining branching stereotypy of lung airways. Development 138, 2359-2368. doi: 10. 1242/dev.060723

Lebeche, D., Malpel, S., and Cardoso, W. V. (1999). Fibroblast growth factor interactions in the developing lung. Mech. Dev. 86, 125-136. doi: 10.1016/ s0925-4773(99)00124-0

Leclerc, K., and Costantini, F. (2016). Mosaic analysis of cell rearrangements during ureteric bud branching in dissociated/reaggregated kidney cultures and in vivo: mosaic analysis using kidney cell cultures. Dev. Dyn. 245, 483-496. doi: $10.1002 /$ dvdy. 24387
Lefevre, J. G., Short, K. M., Lamberton, T. O., Michos, O., Graf, D., Smyth, I. M., et al. (2017). Branching morphogenesis in the developing kidney is governed by rules that pattern the ureteric tree. Development 144, 4377-4385. doi: 10.1242/ dev. 153874

Li, H., Jakobson, M., Ola, R., Gui, Y., Kumar, A., Sipilä, P., et al. (2019). Development of the urogenital system is regulated via the $3^{\prime}$ UTR of GDNF. Sci. Rep. 9:5302.

Lin, Y., Zhang, S., Tuukkanen, J., Peltoketo, H., Pihlajaniemi, T., and Vainio, S. (2003). Patterning parameters associated with the branching of the ureteric bud regulated by epithelial-mesenchymal interactions. Int. J. Dev. Biol. 47, 3-13.

Little, M., Georgas, K., Pennisi, D., and Wilkinson, L. (2010). "Kidney development," in Current Topics in Developmental Biology, ed. R. A. Pedersen (Amsterdam: Elsevier), 193-229.

Liu, Y., Jiang, H., Crawford, H. C., and Hogan, B. L. M. (2003). Role for ETS domain transcription factors Pea3/Erm in mouse lung development. Dev. Biol. 261, 10-24. doi: 10.1016/s0012-1606(03)00359-2

Lu, B. C., Cebrian, C., Chi, X., Kuure, S., Kuo, R., Bates, C. M., et al. (2009). Etv4 and Etv5 are required downstream of GDNF and Ret for kidney branching morphogenesis. Nat. Genet. 41:10.

Lu, P., and Werb, Z. (2008). Patterning mechanisms of branched organs. Science 322, 1506-1509. doi: 10.1126/science. 1162783

Magella, B., Adam, M., Potter, A. S., Venkatasubramanian, M., Chetal, K., Hay, S. B., et al. (2018). Cross-platform single cell analysis of kidney development shows stromal cells express Gdnf. Dev. Biol. 434, 36-47. doi: 10.1016/j.ydbio. 2017.11.006

Majumdar, A., Vainio, S., Kispert, A., McMahon, J., and McMahon, A. P. (2003). Wnt11 and Ret/Gdnf pathways cooperate in regulating ureteric branching during metanephric kidney development. Development 130, 3175-3185. doi: $10.1242 /$ dev.00520

McMahon, A. P. (2016). "Development of the mammalian kidney," in Current Topics in Developmental Biology, ed. R. A. Pedersen (Amsterdam: Elsevier), 31-64. doi: 10.1016/bs.ctdb.2015.10.010

Menshykau, D., Blanc, P., Unal, E., Sapin, V., and Iber, D. (2014). An interplay of geometry and signaling enables robust lung branching morphogenesis. Development 141, 4526-4536. doi: 10.1242/dev.116202

Menshykau, D., and Iber, D. (2013). Kidney branching morphogenesis under the control of a ligand-receptor-based Turing mechanism. Phys. Biol. 10:046003. doi: 10.1088/1478-3975/10/4/046003

Menshykau, D., Kraemer, C., and Iber, D. (2012). Branch mode selection during early lung development. PLoS Comput. Biol. 8:e1002377. doi: 10.1371/journal. pcbi. 1002377

Menshykau, D., Michos, O., Lang, C., Conrad, L., McMahon, A. P., and Iber, D. (2019). Image-based modeling of kidney branching morphogenesis reveals GDNF-RET based Turing-type mechanism and pattern-modulating WNT11 feedback. Nat. Commun. 10:239.

Metzger, R. J., Klein, O. D., Martin, G. R., and Krasnow, M. A. (2008). The branching programme of mouse lung development. Nature 453, 745-750. doi: 10.1038/nature07005

Meyer, T. N., Schwesinger, C., Bush, K. T., Stuart, R. O., Rose, D. W., Shah, M. M., et al. (2004). Spatiotemporal regulation of morphogenetic molecules during in vitro branching of the isolated ureteric bud: toward a model of branching through budding in the developing kidney. Dev. Biol. 275, 44-67. doi: 10.1016/j.ydbio.2004.07.022

Meyer, T. N., Schwesinger, C., Sampogna, R. V., Vaughn, D. A., Stuart, R. O., Steer, D. L., et al. (2006). Rho kinase acts at separate steps in ureteric bud and metanephric mesenchyme morphogenesis during kidney development. Differentiation 74, 638-647. doi: 10.1111/j.1432-0436.2006.00102.x

Michael, L., and Davies, J. A. (2004). Pattern and regulation of cell proliferation during murine ureteric bud development. J. Anat. 204, 241-255. doi: 10.1111/ j.0021-8782.2004.00285.x

Michael, L., Sweeney, D. E., and Davies, J. A. (2005). A role for microfilamentbased contraction in branching morphogenesis of the ureteric bud. Kidney Int. 68, 2010-2018. doi: 10.1111/j.1523-1755.2005.00655.x

Michos, O., Cebrian, C., Hyink, D., Grieshammer, U., Williams, L., D’Agati, V., et al. (2010). Kidney development in the absence of Gdnf and Spryl requires Fgf10. PLoS Genet. 6:e1000809. doi: 10.1371/journal.pgen.1000809

Michos, O. (ed.) (2012). Kidney Development: Methods and Protocols. Springer Protocols 886. New York: Humana Press. 
Min, H., Danilenko, D. M., Scully, S. A., Bolon, B., Ring, B. D., Tarpley, J. E., et al. (1998). Fgf-10 is required for both limb and lung development and exhibits striking functional similarity to Drosophila branchless. Genes Dev. 12, 3156-3161. doi: 10.1101/gad.12.20.3156

Miura, T., and Shiota, K. (2002). Depletion of FGF acts as a lateral inhibitory factor in lung branching morphogenesis in vitro. Mech. Dev. 116, 29-38. doi: 10.1016/s0925-4773(02)00132-6

Moerlooze, L. D., Spencer-Dene, B., Revest, J. M., Hajihosseini, M., Rosewell, I., and Dickson, C. (2000). An important role for the IIIb isoform of fibroblast growth factor receptor 2 (FGFR2) in mesenchymal-epithelial signalling during mouse organogenesis. Development 127, 483-492. doi: 10.1242/dev.127.3.483

Moore, K. A., Polte, T., Huang, S., Shi, B., Alsberg, E., Sunday, M. E., et al. (2005). Control of basement membrane remodeling and epithelial branching morphogenesis in embryonic lung by Rho and cytoskeletal tension. Dev. Dyn. 232, 268-281. doi: 10.1002/dvdy.20237

Moore, M. W., Klein, R. D., Fariñas, I., Sauer, H., Armanini, M., Phillips, H., et al. (1996). Renal and neuronal abnormalities in mice lacking GDNF. Nature 382, 76-79. doi: $10.1038 / 382076 \mathrm{a} 0$

Mujahid, S., Nielsen, H. C., and Volpe, M. V. (2013). MiR-221 and miR-130a regulate lung airway and vascular development. PLoS One 8:e55911. doi: 10. 1371/journal.pone.0055911

Nagalakshmi, V. K., Ren, Q., Pugh, M. M., Valerius, M. T., McMahon, A. P., and Yu, J. (2011). Dicer regulates the development of nephrogenic and ureteric compartments in the mammalian kidney. Kidney Int. 79, 317-330. doi: 10.1038/ ki.2010.385

Nauli, S. M., Jin, X., AbouAlaiwi, W. A., El-Jouni, W., Su, X., and Zhou, J. (2013). Non-motile primary cilia as fluid shear stress mechanosensors. Methods Enzymol. 525, 1-20. doi: 10.1016/b978-0-12-397944-5.00001-8

Nelson, C. M., Gleghorn, J. P., Pang, M.-F., Jaslove, J. M., Goodwin, K., Varner, V. D., et al. (2017). Microfluidic chest cavities reveal that transmural pressure controls the rate of lung development. Development 144, 4328-4335. doi: $10.1242 /$ dev.154823

Nikolić, M. Z., Caritg, O., Jeng, Q., Johnson, J.-A., Sun, D., Howell, K. J., et al. (2017). Human embryonic lung epithelial tips are multipotent progenitors that can be expanded in vitro as long-term self-renewing organoids. eLife 6:e26575.

Nogawa, H., and Ito, T. (1995). Branching morphogenesis of embryonic mouse lung epithelium in mesenchyme-free culture. Development 121, 1015-1022. doi: 10.1242/dev.121.4.1015

Nogawa, H., Morita, K., and Cardoso, W. V. (1998). Bud formation precedes the appearance of differential cell proliferation during branching morphogenesis of mouse lung epithelium in vitro. Dev. Dyn. 213, 228-235. doi: 10.1002/(sici) 1097-0177(199810)213:2<228::aid-aja8>3.0.co;2-i

Ochoa-Espinosa, A., and Affolter, M. (2012). Branching morphogenesis: from cells to organs and back. Cold Spring Harb. Perspect. Biol. 14:a008243. doi: 10.1101/ cshperspect.a008243

Ohuchi, H., Hori, Y., Yamasaki, M., Harada, H., Sekine, K., Kato, S., et al. (2000). FGF10 acts as a major ligand for FGF receptor 2 IIIb in mouse multi-organ development. Biochem. Biophys. Res. Commun. 277, 643-649. doi: 10.1006/ bbrc. 2000.3721

Okubo, T., Knoepfler, P. S., Eisenman, R. N., and Hogan, B. L. (2005). Nmyc plays an essential role during lung development as a dosage-sensitive regulator of progenitor cell proliferation and differentiation. Development 132, 1363-1374. doi: $10.1242 /$ dev. 01678

Onodera, T., Sakai, T., Hsu, J. C., Matsumoto, K., Chiorini, A., and Yamada, K. M. (2010). Btbd7 regulates epithelial cell dynamics and branching morphogenesis. Science 329, 562-565. doi: 10.1126/science. 1191880

Ostrin, E. J., Little, D. R., Gerner-Mauro, K. N., Sumner, E. A., Ríos-Corzo, R., Ambrosio, E., et al. (2018). $\beta$-Catenin maintains lung epithelial progenitors after lung specification. Development 145:dev160788.

Pachnis, V., Mankoo, B., and Costantini, F. (1993). Expression of the c-ret protooncogene during mouse embryogenesis. Development 119, 1005-1017. doi: 10.1242/dev.119.4.1005

Packard, A., Georgas, K., Michos, O., Riccio, P., Cebrian, C., Combes, A. N., et al. (2013). Luminal mitosis drives epithelial cell dispersal within the branching ureteric bud. Dev. Cell 27, 319-330. doi: 10.1016/j.devcel.2013.09.001

Park, W. Y., Miranda, B., Lebeche, D., Hashimoto, G., and Cardoso, W. V. (1998). FGF-10 is a chemotactic factor for distal epithelial buds during lung development. Dev. Biol. 201, 125-134. doi: 10.1006/dbio.1998.8994
Pepicelli, C. V., Kispert, A., Rowitch, D. H., and McMahon, A. P. (1997). GDNF induces branching and increased cell proliferation in the ureter of the mouse. Dev. Biol. 192, 193-198. doi: 10.1006/dbio.1997.8745

Pichel, J. G., Shen, L., Sheng, H. Z., Granholm, A.-C., Drago, J., Grinberg, A., et al. (1996). Defects in enteric innervation and kidney development in mice lacking GDNF. Nature 382, 73-76. doi: 10.1038/382073a0

Pohl, M., Sakurai, H., Bush, K. T., and Nigam, S. K. (2000). Matrix metalloproteinases and their inhibitors regulate in vitro ureteric bud branching morphogenesis. Am. J. Physiol. Renal Physiol. 279, F891-F900.

Prins, G. S., and Putz, O. (2008). Molecular signaling pathways that regulate prostate gland development. Differentiation 76, 641-659. doi: 10.1111/j.14320436.2008.00277.x

Qiao, J., Bush, K. T., Steer, D. L., Stuart, R. O., Sakurai, H., Wachsman, W., et al. (2001). Multiple fibroblast growth factors support growth of the ureteric bud but have different effects on branching morphogenesis. Mech. Dev. 109, 123-135. doi: 10.1016/s0925-4773(01)00592-5

Qiao, J., Sakurai, H., and Nigam, S. K. (1999). Branching morphogenesis independent of mesenchymal- epithelial contact in the developing kidney. Proc. Natl. Acad. Sci. U.S.A. 96, 7330-7335. doi: 10.1073/pnas.96.13.7330

Ramasamy, S. K., Mailleux, A. A., Gupte, V. V., Mata, F., Sala, F. G., Veltmaat, J. M., et al. (2007). Fgf10 dosage is critical for the amplification of epithelial cell progenitors and for the formation of multiple mesenchymal lineages during lung development. Dev. Biol. 307, 237-247. doi: 10.1016/j.ydbio.2007.04.033

Rankin, S. A., Thi Tran, H., Wlizla, M., Mancini, P., Shifley, E. T., Bloor, S. D., et al. (2015). A molecular atlas of Xenopus respiratory system development: molecular Atlas of Xenopus respiratory system development. Dev. Dyn. 244, 69-85.

Resnick, A., and Hopfer, U. (2007). Force-response considerations in ciliary mechanosensation. Biophys. J. 93, 1380-1390. doi: 10.1529/biophysj.107. 105007

Riccio, P., Cebrian, C., Zong, H., Hippenmeyer, S., and Costantini, F. (2016). Ret and Etv4 promote directed movements of progenitor cells during renal branching morphogenesis. PLoS Biol. 14:e1002382. doi: 10.1371/journal.pbio. 1002382

Ritvos, O., Tuuri, T., Erämaa, M., Sainio, K., Hildén, K., Saxén, L., et al. (1995). Activin disrupts epithelial branching morphogenesis in developing glandular organs of the mouse. Mech. Dev. 50, 229-245. doi: 10.1016/0925-4773(94) 00342-k

Rockich, B. E., Hrycaj, S. M., Shih, H. P., Nagy, M. S., Ferguson, M. A. H., Kopp, J. L., et al. (2013). Sox9 plays multiple roles in the lung epithelium during branching morphogenesis. Proc. Natl. Acad. Sci. U.S.A. 110, E4456-E4464.

Rozen, E. J., Schmidt, H., Dolcet, X., Basson, M. A., Jain, S., and Encinas, M. (2009). Loss of sproutyl rescues renal agenesis caused by ret mutation. JASN 20, 255-259. doi: 10.1681/asn.2008030267

Rutledge, E. A., Benazet, J.-D., and McMahon, A. P. (2017). Cellular heterogeneity in the ureteric progenitor niche and distinct profiles of branching morphogenesis in organ development. Development 144, 3177-3188. doi: 10. 1242/dev. 149112

Rutledge, E. A., Parvez, R. K., Short, K. M., Smyth, I. M., and McMahon, A. P. (2019). Morphogenesis of the kidney and lung requires branch-tip directed activity of the Adamts18 metalloprotease. Dev. Biol. 454, 156-169. doi: 10.1016/ j.ydbio.2019.06.012

Saburi, S., Hester, I., Fischer, E., Pontoglio, M., Eremina, V., Gessler, M., et al. (2008). Loss of Fat4 disrupts PCP signaling and oriented cell division and leads to cystic kidney disease. Nat. Genet. 40, 1010-1015. doi: 10.1038/ng.179

Sainio, K., Suvanto, P., Davies, J., Wartiovaara, J., Wartiovaara, K., Saarma, M., et al. (1997). Glial-cell-line-derived neurotrophic factor is required for bud initiation from ureteric epithelium. Development 124, 4077-4087. doi: 10.1242/dev.124. 20.4077

Sakai, T., Larsen, M., and Yamada, K. M. (2003). Fibronectin requirement in branching morphogenesis. Nature 423:6.

Sakakura, T., Nishizuka, Y., and Dawe, C. J. (1976). Mesenchyme-dependent morphogenesis and epithelium-specific cytodifferentiation in mouse mammary gland. Science 194, 1439-1441. doi: 10.1126/science.827022

Sánchez, M. P., Silos-Santiago, I., Frisén, J., He, B., Lira, S. A., and Barbacid, M. (1996). Renal agenesis and the absence of enteric neurons in mice lacking GDNF. Nature 382, 70-73. doi: 10.1038/382070a0 
Sanna-Cherchi, S., Sampogna, R. V., Papeta, N., Burgess, K. E., Nees, S. N., Perry, B. J., et al. (2013). Mutations in DSTYK and dominant urinary tract malformations. N. Engl. J. Med. 369, 621-629.

Sarin, S., Boivin, F., Li, A., Lim, J., Svajger, B., Rosenblum, N. D., et al. (2014). $\beta$ catenin overexpression in the metanephric mesenchyme leads to renal dysplasia genesis via cell-autonomous and non-cell-autonomous mechanisms. Am. J. Pathol. 184, 1395-1410. doi: 10.1016/j.ajpath.2014.01.018

Schittny, J. C. (2017). Development of the lung. Cell Tissue Res. 367, 427-444.

Schnatwinkel, C., and Niswander, L. (2013). Multiparametric image analysis of lung-branching morphogenesis. Dev. Dyn. 16, 622-637. doi: 10.1002/dvdy. 23961

Schuchardt, A., D’Agati, V., Larsson-Blomberg, L., Costantini, F., and Pachnis, V. (1994). Defects in the kidney and enteric nervous system of mice lacking the tyrosine kinase receptor Ret. Nature 367, 380-383. doi: 10.1038/367380a0

Schuchardt, A., D’Agati, V., Pachnis, V., and Costantini, F. (1996). Renal agenesis and hypodysplasia in ret-k- mutant mice result from defects in ureteric bud development. Development 122, 1919-1929. doi: 10.1242/dev.122.6.1919

Schuger, L., Skubitz, O., and Chang, V. (1991). Identification of laminin domains involved in branching morphogenesis: effects of anti-laminin monoclonal antibodies on mouse embryonic lung development. Dev. Biol. 146, 531-541. doi: 10.1016/0012-1606(91)90254-z

Sekine, K., Ohuchi, H., Fujiwara, M., Yamasaki, M., Yoshizawa, T., Sato, T., et al. (1999). Fgf10 is essential for limb and lung formation. Nat. Genet. 21, 138-141. doi: $10.1038 / 5096$

Shah, M. M. (2004). Branching morphogenesis and kidney disease. Development $131,1449-1462$.

Shakya, R., Jho, E., Kotka, P., Wu, Z., Kholodilov, N., Burke, R., et al. (2005a). The role of GDNF in patterning the excretory system. Dev. Biol. 283, 70-84. doi: 10.1016/j.ydbio.2005.04.008

Shakya, R., Watanabe, T., and Costantini, F. (2005b). The role of GDNF/Ret signaling in ureteric bud cell fate and branching morphogenesis. Dev. Cell 8, 65-74. doi: 10.1016/j.devcel.2004.11.008

Shannon, J. M., McCormick-Shannon, K., Burhans, M. S., Shangguan, X., Srivastava, K., and Hyatt, B. A. (2003). Chondroitin sulfate proteoglycans are required for lung growth and morphogenesis in vitro. Am. J. Physiol. Lung Cell. Mol. Physiol. 285, L1323-L1336.

Shao, X., Li, Q., Mogilner, A., Bershadsky, A. D., and Shivashankar, G. V. (2015). Mechanical stimulation induces formin-dependent assembly of a perinuclear actin rim. Proc. Natl. Acad. Sci. U.S.A. 112, E2595-E2601.

Shifley, E. T., Kenny, A. P., Rankin, S. A., and Zorn, A. M. (2012). Prolonged FGF signaling is necessary for lung and liver induction in Xenopus. BMC Dev. Biol. 12:27. doi: 10.1186/1471-213X-12-27

Short, K., Hodson, M., and Smyth, I. (2013). Spatial mapping and quantification of developmental branching morphogenesis. Development 140, 471-478. doi: 10.1242/dev.088500

Short, K. M., Combes, A. N., Lefevre, J., Ju, A. L., Georgas, K. M., Lamberton, T., et al. (2014). Global quantification of tissue dynamics in the developing mouse kidney. Dev. Cell 29, 188-202. doi: 10.1016/j.devcel.2014.02.017

Short, K. M., Hodson, M. J., and Smyth, I. M. (2010). Tomographic quantification of branching morphogenesis and renal development. Kidney Int. 77, 1132-1139. doi: $10.1038 / \mathrm{ki} .2010 .42$

Short, K. M., and Smyth, I. M. (2016). "Quantification of developmental branching morphogenesis," in Kidney Development, Disease, Repair and Regeneration, ed. M. H. Little (Amsterdam: Elsevier), 57-65. doi: 10.1016/b978-0-12-800102-8. 00005-9

Simonet, W. S., DeRose, M. L., Bucay, N., Nguyen, H. Q., Wert, S. E., Zhou, L., et al. (1995). Pulmonary malformation in transgenic mice expressing human keratinocyte growth factor in the lung. Proc. Natl. Acad. Sci. U.S.A. 92, 1246112465. doi: 10.1073/pnas.92.26.12461

Sims-Lucas, S., Argyropoulos, C., Kish, K., McHugh, K., Bertram, J. F., Quigley, R., et al. (2009). Three-dimensional imaging reveals ureteric and mesenchymal defects in Fgfr2 -mutant kidneys. JASN 20, 2525-2533. doi: 10.1681/asn. 2009050532

Spooner, B. S., and Wessells, N. K. (1970). Mammalian lung development: interactions in primordium formation and bronchial morphogenesis. J. Exp. Zool. 175:10.

Spurlin, J. W., and Nelson, C. M. (2016). Building branched tissue structures: from single cell guidance to coordinated construction. Philod. Trans. R. Soc. 372:20150527. doi: 10.1098/rstb.2015.0527
Srinivas, S., Wu, Z., Chen, C. M., D’Agati, V., and Costantini, F. (1999). RET signaling and ureteric bud development. Development 126, 1375-1386. doi: 10.1242/dev.126.7.1375

Sternlicht, M. D., Kouros-Mehr, H., Lu, P., and Werb, Z. (2006). Hormonal and local control of mammary branching morphogenesis. Differentiation 74, 365-381. doi: 10.1111/j.1432-0436.2006.00105.x

Stopka, A., Kokic, M., and Iber, D. (2019). Cell-based simulations of biased epithelial lung growth. Phys. Biol. 17:016006. doi: 10.1088/1478-3975/ab5613

Tang, M.-J., Cai, Y., Tsai, S.-J., Wang, Y.-K., and Dressler, G. R. (2002). Ureteric bud outgrowth in response to RET activation is mediated by phosphatidylinositol 3-kinase. Dev. Biol. 243, 128-136. doi: 10.1006/dbio.2001.0557

Tang, M.-J., Worley, D., Sanicola, M., and Dressler, G. R. (1998). The RET-glial cell-derived neurotrophic factor (GDNF) pathway stimulates migration and chemoattraction of epithelial cells. J. Cell Biol. 142, 1337-1345. doi: 10.1083/ jcb.142.5.1337

Tang, N., Marshall, W. F., McMahon, M., Metzger, R. J., and Martin, G. R. (2011). Control of mitotic spindle angle by the RAS-Regulated ERK1/2 pathway determines lung tube shape. Science 333, 342-345. doi: 10.1126/science. 1204831

Tichelaar, J. W., Lu, W., and Whitsett, J. A. (2000). Conditional expression of fibroblast growth factor-7 in the developing and mature lung. J. Biol. Chem. 275:8.

Towers, P. R., Woolf, A. S., and Hardman, P. (1998). Glial cell line-derived neurotrophic factor stimulates ureteric bud outgrowth and enhances survival of ureteric bud cells in vitro. Exp. Nephrol. 6, 337-351. doi: 10.1159/000020541

Treanor, J. J. S., Goodman, L., de Sauvage, F., Stone, D. M., Poulsen, K. T., Beck, C. D., et al. (1996). Characterization of a multicomponent receptor for GDNF. Nature 382, 80-83. doi: 10.1038/382080a0

Trueb, B., Amann, R., and Gerber, S. D. (2013). Role of FGFRL1 and other FGF signaling proteins in early kidney development. Cell. Mol. Life Sci. 70, 2505-2518. doi: 10.1007/s00018-012-1189-9

Turing, A. (1952). The chemical basis of morphogenesis. Philos. Trans. R. Soc. Lond. 36, 153-197.

Unbekandt, M., del Moral, P.-M., Sala, F. G., Bellusci, S., Warburton, D., and Fleury, V. (2008). Tracheal occlusion increases the rate of epithelial branching of embryonic mouse lung via the FGF10-FGFR2b-Sprouty2 pathway. Mech. Dev. 125, 314-324. doi: 10.1016/j.mod.2007.10.013

Varner, V. D., Gleghorn, J. P., Miller, E., Radisky, D. C., and Nelson, C. M. (2015). Mechanically patterning the embryonic airway epithelium. Proc. Natl. Acad. Sci. U.S.A. 112, 9230-9235. doi: 10.1073/pnas.1504102112

Villasenor, A., Chong, D. C., Henkemeyer, M., and Cleaver, O. (2010). Epithelial dynamics of pancreatic branching morphogenesis. Development 137, 42954305. doi: 10.1242/dev.052993

Volckaert, T., Campbell, A., Dill, E., Li, C., Minoo, P., and De Langhe, S. (2013). Localized Fgf10 expression is not required for lung branching morphogenesis but prevents differentiation of epithelial progenitors. Development 140, 37313742. doi: 10.1242/dev.096560

Volpe, M., Ramadurai, V., and Nielsen, P. (2007). HoxB-5 down regulation alters Tenascin-C, FGF10 AND HoxB gene expression patterns in pseudoglandular period fetal mouse lung. Front. Biosci. 12, 860-873. doi: 10.2741/2108

Wang, J., Ito, T., Udaka, N., Okudela, K., Yazawa, T., and Kitamura, H. (2005). PI3K-AKT pathway mediates growth and survival signals during development of fetal mouse lung. Tissue Cell 37, 25-35. doi: 10.1016/j.tice.2004.09.002

Wang, S., Sekiguchi, R., Daley, W. P., and Yamada, K. M. (2017). Patterned cell and matrix dynamics in branching morphogenesis. J. Cell Biol. 216, 559-570. doi: $10.1083 /$ jcb. 201610048

Warburton, D., Bellusci, S., De Langhe, S., Del Moral, P.-M., Fleury, V., Mailleux, A., et al. (2005). Molecular mechanisms of early lung specification and branching morphogenesis. Pediatr. Res. 57, 26R-37R.

Warburton, D., El-Hashash, A., Carraro, G., Tiozzo, C., Sala, F., Rogers, O., et al. (2010). "Lung organogenesis," in Current Topics in Developmental Biology, ed. R. A. Pedersen (Amsterdam: Elsevier), 73-158.

Watanabe, T., and Costantini, F. (2004). Real-time analysis of ureteric bud branching morphogenesis in vitro. Dev. Biol. 271, 98-108. doi: 10.1016/j.ydbio. 2004.03.025

Weaver, M., Dunn, N. R., and Hogan, B. L. M. (2000). Bmp4 and Fgf10 play opposing roles during lung bud morphogenesis. Development 127, 2695-2704. doi: $10.1242 / \mathrm{dev} \cdot 127.12 .2695$ 
Lang et al.

Organ-Specific Branching Morphogenesis

Weibel, E. R. (1991). Fractal geometry: a design principle for living organisms. Am. J. Physiol. Lung Cell. Mol. Physiol. 261, L361-L369.

Weinbaum, S., Duan, Y., The, M. M., and You, L. (2011). An integrative review of mechanotransduction in endothelial, epithelial (Renal) and dendritic cells (Osteocytes). Cell. Mol. Bioeng. 4, 510-537. doit: 10.1007/s12195-011-0179-6

Weasels, N. K., and Cohen, J. H. (1968). Effects of collagenase on developing epithelia in vito: lung, ureteric bud, and pancreas. Lev. Biol. 18, 294-309. dor: 10.1016/0012-1606(68)90037-7

White, A. C., Xu, J., Yin, Y., Smith, C., Schmid, G., and Ornitz, D. M. (2006). FGF9 and SHH signaling coordinate lung growth and development through regulation of distinct mesenchymal domains. Development 133, 1507-1517. doa: $10.1242 /$ dev.02313

Yates, L. L., Schnatwinkel, C., Murdoch, J. N., Bogani, D., Formstone, C. J., Townsend, S., et al. (2010). The PCP genes Celsr1 and Vangl2 are required for normal lung branching morphogenesis. Hum. Mol. Genet. 19, 2251-2267. dor: $10.1093 / \mathrm{hmg} / \mathrm{ddq} 104$

Yin, Y., and Ornitz, D. M. (2020). FGF9 and FGF10 activate distinct signaling pathways to direct lung epithelial specification and branching. Sci. Signal. 13:eaay4353. do: 10.1126/scisignal.aay4353

Yin, Y., Wang, F., and Ornitz, D. M. (2011). Mesothelial- and epithelialderived FGF9 have distinct functions in the regulation of lung development. Development 138, 3169-3177. dor: 10.1242/dev.065110

Young, R. E., Jones, M.-K., Hines, E. A., Li, R., Luo, Y., Shi, W., et al. (2020). Smooth muscle differentiation is essential for airway size, tracheal cartilage segmentation, but dispensable for epithelial branching. Nev. Cell 53, 73.e585.e5.

Ya, J. (2014). miRNAs in mammalian ureteric bud development. Pediatr. Nephrol. 29, 745-749. do: 10.1007/s00467-013-2734-y

Yuan, T., Volckaert, T., Chanda, D., Thannickal, V. J., and De Langhe, S. P. (2018). Fgf10 signaling in lung development, homeostasis, disease, and repair after injury. Front. Genet. 9:418. do: 10.3389/fgene.2018.00418

Zhao, H., Kegs, H., Grady, S., Truong, H.-T., Robinson, M. L., Baum, M., et al. (2004). Role of fibroblast growth factor receptors 1 and 2 in the ureteric bud. Lev. Biol. 276, 403-415. dor: 10.1016/j.ydbio.2004.09.002

Zhu, W., and Nelson, C. M. (2012). PI3K signaling in the regulation of branching morphogenesis. Biosystems 109, 403-411. doa: 10.1016/j.biosystems.2012.04. 004

Conflict of Interest: The authors declare that the research was conducted in the absence of any commercial or financial relationships that could be construed as a potential conflict of interest.

Copyright (C) 2021 Lang, Conrad and lIber. This is an open-access article distributed under the terms of the Creative Commons Attribution License (CC BY). The use, distribution or reproduction in other forums is permitted, provided the original authors) and the copyright owners) are credited and that the original publication in this journal is cited, in accordance with accepted academic practice. No use, distribution or reproduction is permitted which does not comply with these terms.

Frontiers in Cell and Developmental Biology | www.frontiersin.org

17

June 2021 | Volume 9 | Article 671402 